\title{
Lost in modelling and simulation?
}

\author{
Kiyohiko Sugano
}

Molecular Pharmaceutics Lab., College of Pharmaceutical Sciences, Ritsumeikan University, 1-1-1, Noji-higashi, Kusatsu, Shiga 525-8577, Japan

E-mail: suganok@fc.ritsumei.ac.jp; Tel.: +81-77-561-2773

Received: November 06, 2021; Revised: March 05, 2021; Published: March 22, 2021

\begin{abstract}
Over the past few decades, physiologically-based pharmacokinetic modelling (PBPK) has been anticipated to be a powerful tool to improve the productivity of drug discovery and development. However, recently, multiple systematic evaluation studies independently suggested that the predictive power of current oral absorption (OA) PBPK models needs significant improvement. There is some disagreement between the industry and regulators about the credibility of OA PBPK modelling. Recently, the editorial board of AMDET\&DMPK has announced the policy for the articles related to PBPK modelling (Modelling and simulation ethics). In this feature article, the background of this policy is explained: (1) Requirements for scientific writing of PBPK modelling, (2) Scientific literacy for PBPK modelling, and (3) Middle-out approaches. PBPK models are a useful tool if used correctly. This article will hopefully help advance the science of OA PBPK models.
\end{abstract}

(C)2021 by the authors. This article is an open-access article distributed under the terms and conditions of the Creative Commons Attribution license (http://creativecommons.org/licenses/by/4.0/).

\section{Keywords}

physiologically-based pharmacokinetic modelling; scientific literacy; transparency; reproducibility; middleout approach; structural identifiability

\section{Introduction}

Over the past few decades, physiologically-based pharmacokinetic modelling (PBPK) has been anticipated to be a powerful tool to improve the productivity of drug discovery and development. Several sophisticated software products have been commercialized since the late 1990s. Plenty of case study reports have been published in peer-reviewed journals, showing nearly perfect prediction, prediction error being much smaller than the variation in the clinical plasma concentration $\left(C_{p}\right)$ - time profile. It seems that we already have achieved a "prediction paradise"[1]. ...Really? Recently, multiple systematic evaluation studies independently suggested that the "bottom-up" predictive power of current oral absorption (OA) PBPK models needs significant improvement [2-6]. Almost all case studies had to use parameter optimization on a drug-by-drug basis to fit the simulated plasma concentration $\left(C_{p}\right)$ - time curve to clinical data (Part 3, section 3.4). Expert scientists continue hard experimental works to better understand in vivo systems and improve the predictive performance of in vitro systems [7-9]. There is some disagreement between the industry and regulators about the credibility of PBPK modelling $[10,11]$. "Publication bias" (Part 2, section 2.4) and "parameter optimization" (Part 3) have been identified as the main issues of the 
case studies [11]. Recently, a more realistic view about PBPK modelling has been reported by an industrial consortium [12]. Are we lost in modelling and simulation?

Recently, the editorial board of $A M D E T$ and $D M P K$ has announced the policy for the articles related to PBPK modelling (Modelling and simulation ethics). This policy is introduced to enhance the science of PBPK modelling. In this article, the background of this policy is explained. This article consists of three parts:

Part 1: Requirements for scientific writing of PBPK modelling,

Part 2: Scientific literacy for PBPK modelling,

Part 3: Middle-out approach (parameter back-calculation from clinical PK data).

The topics discussed in this article have been repeatedly raised before. Transparency and reproducibility (calculation traceability) are critically important for scientific writing (Part 1) [12-14]. From the perspective of evidence-based medicine, case studies are less reliable for assessing the predictive power of a model (Part 2) [15-17]. The issue of parameter identifiability in mathematical modelling has been repeatedly warned in the literature (Part 3) [18-24].

In this article, the above points will be discussed focusing on OA PBPK modelling. But this article would also be beneficial to the other PBPK models. PBPK models will be a useful tool if used correctly. This article will hopefully enhance the science of OA PBPK modelling in the future.

\section{Part 1: Requirements for scientific writing of PBPK modelling}

The policy on the scientific writing of physiologically-based pharmacokinetic modelling (PBPK) articles complements the current journal's author guidelines that cover in vivo and in vitro methods based on scientific literacy. This guideline is also in line with regulatory guidance for industry regarding PBPK modelling [12-14]. In this article, past articles that do not comply with this policy are not quoted, because it would be disadvantageous for the authors. However, readers will see that the majority of the past papers have some issues raised in this article.

Transparency and reproducibility are mandatory to ensure the credibility of PBPK modelling. As sciences and technology progress, model equations and physiological data can be updated in the future (section 2.1). Therefore, the use of the current best estimate parameter in PBPK modelling is appropriate. However, if the physiological data or model equation changes in the future, past articles need to be re-evaluated. For example, a wide variety of small intestinal fluid volume $\left(V_{\text {si }}\right)$ has been used in oral absorption (OA) PBPK modelling, ranging from less than $100 \mathrm{~mL}$ to over $1500 \mathrm{~mL}$ (the former is based on the recent MRI measurements) [25-29]. Despite more than a 15-fold difference, they all claimed good predictability (section 3.3). This cannot be true. If all details had been reported, we can trace the calculation and retrospectively inspect the reason for this contradiction. But if there was an undisclosed part, it is not possible to judge whether the past good prediction is just a lucky coincidence or due to other reasons. When such an inconsistency arises in regulatory submissions, it causes a more troubling situation. To ensure the credibility of PBPK modelling, authors must write the manuscript as transparent as possible to enable inspection by peer-reviewers and ensure reproducibility by independent third parties.

\subsection{Introduction section}

The purpose of PBPK modelling should be explained in the introduction section. The question of interest and the context of use (COU) for PBPK modelling should be described [10]. There is no one-size-fits-all model (section 2.8). A mathematical model that suits the purpose should be selected. The reason for selecting a PBPK model should be explained. 


\subsection{Method section}

Based on the scientific ethics of transparency and reproducibility, authors are requested to disclose the model equations, physiological parameters, and drug parameters (section 2.1) that are sufficient for peerreview (or appropriate references to them). Undisclosed parts cannot be peer-reviewed in the first place. In addition, according the rule of science, an experimental section should be written as detailed as possible so that to "enable" reproducing the same result. This point is critical for ensuring the credibility of science. If the word "reproducibility" is interpreted literally, even if the model equations and parameters are not disclosed (as in a black box model), the results can be reproduced by using the same input data and the same software (same version). In the context of scientific credibility, for the articles of mechanistic models (including PBPK models (section 2.1)), it should be taken as calculation traceability. A method section should be detailed enough to enable someone who wants to trace the calculation process, at least in the essential parts for COU. If a part of the model is not disclosed, that part becomes a "black box". Because a black box can mask errors in any part of the model, the credibility of the entire research is damaged by the existence of only one undisclosed part.

The scientific validity of a research article is the author's responsibility, even when using commercial software. In papers using commercial software, the model equations and physiological parameters are often described as "default". The default information may have been disclosed in a user's manual. However, it is not available for peer-reviewers and readers. The default model equations and physiological parameters must also be publicly disclosed.

In nearly all case study reports of OA PBPK modelling, case-by-case parameter optimization (backcalculation) has been committed to fit the simulated plasma concentration $\left(C_{p}\right)$ - time curve to the clinical observation on a drug-by-drug basis (called "local middle-out approach", see Part 3). Any parameter backcalculation from clinical PK data must be explicitly stated in the method section. Case-by-case parameter back-calculation could have been unconsciously committed. For example, ad hoc selection of estimated permeability values from in silico, in vitro, or in situ data is a kind of parameter back-calculation. Various terms implying a subtle adjustment such as "optimize", "fit", "adjust", "recover", "refine", and "software estimated", have been used to refer to parameter back-calculation. However, the difference from the initial value often exceeds two-fold due to the large errors in in vitro - in vivo extrapolation (IVIVE) and/or in silico prediction [30,31] (section 3.10).

When a parameter is calculated from a chemical structure by an in silico model, the prediction accuracy of the in silico model should be shown (or referenced) (section 3.8 (iv)).

\subsection{Results section}

When case-by-case parameter back-calculation was committed, the initial input parameters and the simulation results before parameter back-calculation must be reported in the result section (Figure 1). Unfortunately, the failed prediction is often undisclosed in case study reports. However, this information is important for evaluating the creditability of a back-calculated parameter. For the advancement of science, failed predictions are just as (or even more) important than successful cases (section 3.4).

After case-by-case parameter back-calculation, the fitted curve must be labeled as "fitted" (NOT "predicted") for the same clinical PK data used for parameter fitting. The fitted $C_{p}$ - time curve is not a predicted curve, because the observed $C_{p}$ - time profile has been used to back-calculate the parameter (self-referencing) (section 3.11).

In oral absorption (OA) PBPK modelling, the fraction of a dose absorbed $\left(F_{\mathrm{a}}\right)$ is one of the most 
important outputs for understanding the oral absorption of a drug. Although clinical $F_{\mathrm{a}}$ data (or its surrogates (section 2.6)) is not always available for model validation, simulated $F_{\mathrm{a}}$ data is important for interpreting the simulation results regarding the oral absorption of a drug, at least for biopharmaceutics and formulation scientists (section 2.2). Therefore, a simulated $F_{\mathrm{a}}$ value should be reported. The simulated $F_{\mathrm{a}}$ value (or the $F_{\mathrm{a}}$ - time profile) is available as an output in all commercial OA PBPK software products. However, unfortunately, this value has not been reported in many reports.

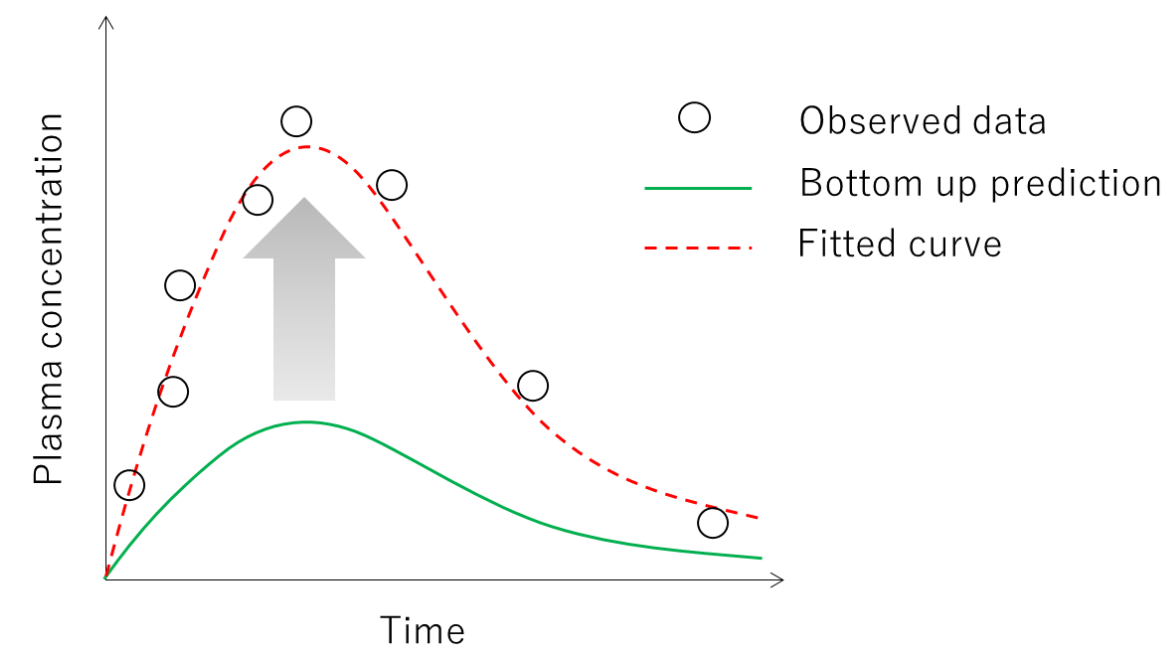

Figure 1. Schematic illustration of simulated and observed $C_{p}$ - time profiles

\subsection{Discussion section}

When parameter back-calculation from clinical PK data is committed, the following points should be discussed (See Part 3 for details) [18-24,32-34]: (i) the reason for the mismatch of the initial "bottom-up" prediction and clinical PK data, (ii) the reason for selecting a parameter as the subject of back-calculation (sections 3.5 to 3.7), (iii) parameter identifiability (section 3.2), (iv) the accuracy of the other parameters than the subject of parameter back-calculation (section 3.8), (v) the plausibility of back-calculated parameter considering physicochemical properties, in vitro data, and in vivo physiology (section 3.10), (vi) the constancy of the back-calculated parameter in the clinical and/or population conditions (COU) to be predicted (sections 3.3 and 3.13), (vii) the validity of the optimized model (section 3.11).

\subsection{References}

The above policy applies to the references. The authors should cite reliable articles as references.

\section{Part 2: Scientific literacy for physiologically-based pharmacokinetic modelling}

In Part 1, we discussed how to write a scientific article for PBPK modeling. In this part, we will discuss how to interpret PBPK papers and how to evaluate PBPK models. Physiologically-based pharmacokinetic (PBPK) models are not as easy to use as a smartphone app. As mentioned in the introduction, commercial PBPK software may not be so perfect as a user might believe $[2-6,11,35,36]$. Before using a PBPK model, we must understand the scientific literacy for mathematical modelling. In this part, the scientific literacy required for PBPK modelling is discussed. We are fully aware that there are various opinions among the modelers on this topic. The purpose of this part is to suggest several viewpoints when using a PBPK model.

\subsection{The basic concept of physiologically-based pharmacokinetic models}

Basically, a PBPK model consists of independent a priori information of drug and formulation parameters, physiological and biological parameters at the organ level, and model equations [37]. All model 
equations and parameters have a physical and physiological basis (mechanistic basis) (Figure 2). PBPK models provide a mechanistic representation of pharmacokinetics and allow a priori "bottom-up" prediction of in vivo PK profiles from in vitro data for various clinical situations. To simulate the effect of a physiological factor, the model equation must include the factor as a system parameter. As drug parameters, the parameters that are intrinsic to a drug should be used. By combining drug-intrinsic parameters with physiological factors in model equations, the effects of physiological conditions can be simulated. This model structure allows the PBPK model to handle population variability and physiological covariates. Furthermore, the oral absorption (OA) PBPK model can account for the bioequivalence of various formulations considering confidence intervals. This point is one of the specific features that can be handled by PBPK modelling.

(A)
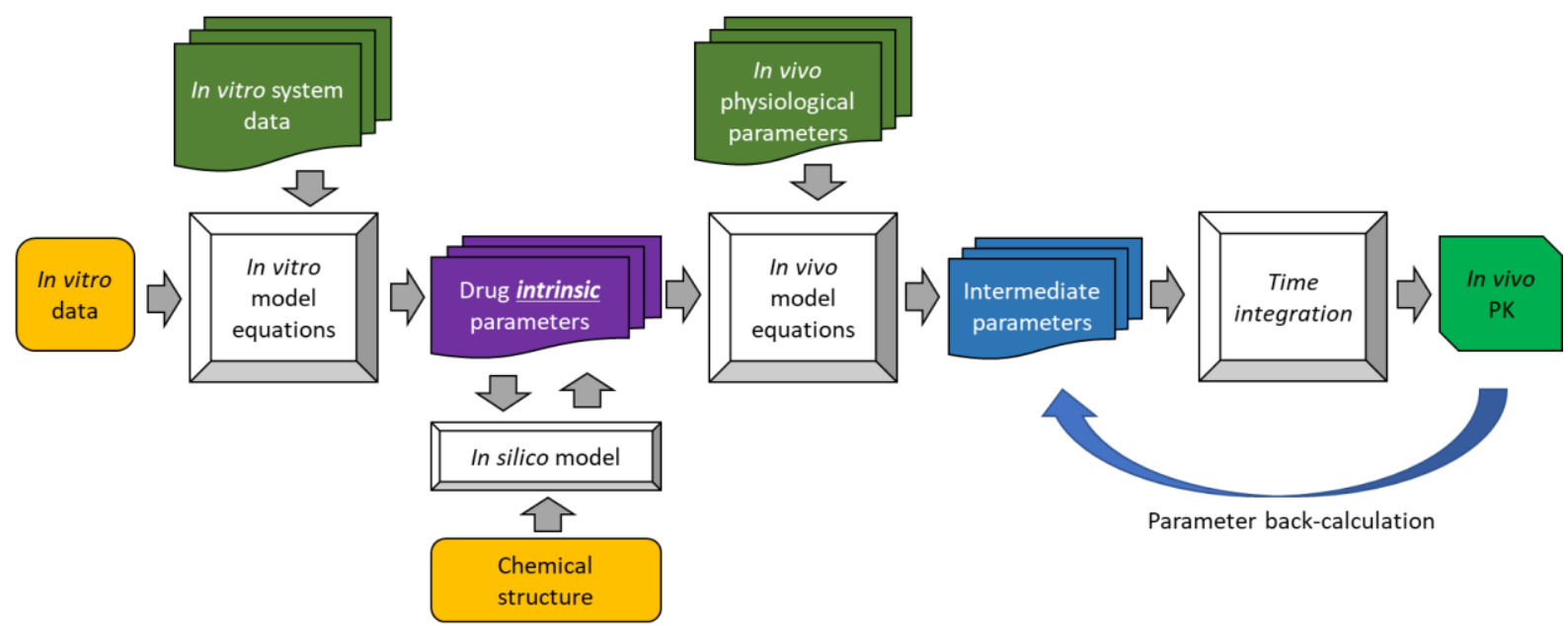

(B)

$$
S=S_{0}\left(1+\frac{K_{a}}{\left[H^{+}\right]}+\frac{K_{b m, 0}\left[C_{b m}\right]}{\left[H_{2} O\right]}+\frac{K_{a}}{\left[H^{+}\right]} \frac{K_{b m,-}\left[C_{b m}\right]}{\left[H_{2} O\right]}\right)
$$

Figure 2. Overall prediction scheme of PBPK modelling $(A)$ and the solubility model in biorelevant media for an acidic drug (B). (A) In vitro data is first reduced to drug intrinsic parameters using the mathematical model of an in vitro system [38]. The drug intrinsic parameters are then converted to in vivo PK profiles via intermediate parameters (e.g., the permeation rate constant $\left.\left(k_{\text {perm }}\right)\right)$. The drug intrinsic parameters are directly related to a chemical structure so that suitable for in silico prediction and drug design. (B) Drug intrinsic parameters: purple, physiological parameters: green, and intermediate parameter: blue $[39,40] . K_{\mathrm{a}}$ is the dissociation constant, $S_{0}$ is the intrinsic solubility of a drug, and $K_{\mathrm{bm}, 0}$ and $K_{\mathrm{bm},-}$ are the bile micelle partition coefficients for unionized and anionic drug molecular species, respectively. $\left[C_{\mathrm{bm}}\right]$ is the bile micelle concentration, $\left[\mathrm{H}^{+}\right]$is the proton concentration $\left(=10^{-\mathrm{pH}}\right)$, and $\left[\mathrm{H}_{2} \mathrm{O}\right]$ is the concentration of water.

An OA PBPK model consists of the model equations of solubility, dissolution rate, permeability, etc. Therefore, it is important to understand these equations before using PBPK modelling (section 1.2, 2.3, and 2.7). For example, in a physiologically-based solubility model (Figure 2B), the $\mathrm{pH}$ and the bile micelle concentration $\left(C_{\mathrm{bm}}\right)$ are used as physiological parameters, and the intrinsic solubility $\left(S_{0}\right), \mathrm{p} K_{\mathrm{a}}$, and bile micelle partition coefficients $\left(K_{\mathrm{bm}}\right)$ (for each unionized and ionized drug molecules) are used as drug parameters [35,39,40]. Physiologically-based dissolution [41-48] and permeation [49-55] models have already been reported in the literature and implemented in some OA PBPK models. The followings are examples of physiologically-based dissolution and permeation models in the simplest form. 


\section{Dissolution model (for mono-dispersed spherical small particles) [41-48,56]}

$$
\frac{\mathrm{d} X_{\text {undissolv }}}{\mathrm{d} t}=-\frac{3 D_{\text {eff }} S_{\text {surface }}}{\rho r_{\mathrm{p}}^{2}} X_{\text {undissolv }}^{1 / 3} \operatorname{Dose}^{2 / 3}\left(1-\frac{X_{\text {dissolv }}}{S_{\text {dissolv }} V_{G l}}\right)
$$

where $X_{\text {undissolv }}$ and $X_{\text {dissolv }}$ are the amounts of a drug undissolved and dissolved in the gastrointestinal fluid, respectively. $D_{\text {eff }}$ is the effective diffusion coefficient, Dose is the dose strength, $\rho$ is the true density of the drug substance, and $r_{\mathrm{p}}$ is the particle radius $\left(r_{\mathrm{p}}<30 \mu \mathrm{m}\right) . S_{\text {surface }}$ and $S_{\text {dissolv }}$ are the solubilities of a drug at the particle surface and in the bulk fluid, respectively. $V_{G I}$ is the gastrointestinal fluid volume. The dissolution rate constant $\left(k_{\text {diss }}\right)$ is $k_{\text {diss }}=3 D_{\text {eff }} S_{\text {surface }} /\left(\rho r_{\mathrm{p}}{ }^{2}\right) . S_{\text {surface, }} S_{\text {dissolv, }}$ and $D_{\text {eff }}$ are affected by physiological factors such as $\mathrm{pH}$, buffer capacity, bile micelle concentration (Figure 1(b)).

\section{Permeation model (for passive diffusion) [49-55]}

$$
\begin{aligned}
& \frac{\mathrm{d} X_{\text {perm }}}{\mathrm{d} t}=k_{\text {perm }} X_{\text {dissolv }}=\frac{2 D F}{R_{\mathrm{Gl}}} P_{\text {eff }} X_{\text {dissolv }} \\
& P_{\text {eff }}=\frac{P E}{\frac{1}{V E \cdot f_{\mathrm{u}}\left(f_{0} P_{\text {trans } 0}+P_{\text {para }}\right)}+\frac{1}{\frac{D_{\text {eff }}}{h_{\text {eff }}}+P_{\mathrm{WC}}}}
\end{aligned}
$$

where $X_{\text {perm }}$ is the amounts of a drug permeated the intestinal wall, $k_{\text {perm }}$ is the permeation rate constant, $D F$ is the degree of flatness of the small intestinal tube, $R_{\mathrm{Gl}}$ is the radius, $P_{\text {eff }}$ is the effective intestinal membrane permeability, $P E$ is the plica expansion factor, $P_{\text {UWL }}$ is the UWL permeability, $V E$ is the villi expansion factor, $f_{\mathrm{u}}$ is the fraction of unbound drug molecule species (free fraction), $f_{0}$ is the fraction of undissociated drug molecule species (calculated from $\mathrm{p} K_{\mathrm{a}}$ and $\mathrm{pH}$ ), $P_{\text {transo }}$ is the intrinsic passive transcellular permeability, $P_{\text {para }}$ is the paracellular permeability, $h_{\text {eff }}$ is the effective thickness of the UWL, and $P_{\mathrm{wc}}$ is the water conveyance.

Instead of using a physiologically-based mechanistic dissolution model, an experimental in vitro dissolution profile can be plugged into an OA PBPK model as a kind of intermediate parameter (Figure 2A)[57]. This strategy is often used when the dissolution process is the key determinant of oral drug absorption. In this case, the dissolution profile is pre-fixed so that it is not altered by the physiological factors in the computer simulation. For example, when a dissolution profile measured at $\mathrm{pH} 1.2$ is plugged into an OA PBPK model, it is not changed even if the gastric $\mathrm{pH}$ value is changed from $\mathrm{pH} 1.2$ to $\mathrm{pH} 5.0$ in the OA PBPK model. Consequently, it cannot account for the effect of inter- or intra- subject variability of relevant physiological parameters on drug dissolution processes. Such a simulation strategy may be understood as a variation of the convolution method, rather than "physiologically-based" computational modelling (in the case of using in vivo predictive dissolution testing [58-61], the in vitro dissolution profile itself is physiologically-based).

\subsection{To Explain or to predict? Which is the position of a PBPK model?}

The purpose of mathematical modeling is not only to calculate the predicted values, but also to explain the observed and predicted data (understanding, interpretation) [62]. The interpretability of a model is especially important for medical applications. For an explanation-oriented model, ideally, all system parameters should be set based on independent a priori information. From the viewpoint of mathematical modelling, PBPK models are more of an explanation-oriented model when compared to more complex statistic models like deep learning [63]. In general, the interpretability of a model decreases as the model 
becomes more complex. Simple models have a great value of "interpretability" by themselves [64]. On the other hand, complex models may (or may not) show better predictive power (prediction performance, generalization performance) (section 2.8).

The predictive power and description capability of a model are different. The description capability of a model is to describe existing data, whereas the predictive power is to predict unknown data. As a model becomes more complex and flexible, description capability always increases. However, predictive power does not always increase, because assumptions and errors can accumulate with the increasing complexity of a model (sections 2.8 and 3.10). When parameterization is used, a complex model is often prone to overfitting (Figure 3).

In mathematical modelling, generally speaking, to compensate for its lesser interpretability and its higher risk of overfitting, a complex model should show a significant advantage over a simple model with respect to predictive power for the context of use [20,64-67]. If simple and complex models show comparable predictive power with regard to the context of use, the simple model should be selected (cf. Occam's razor, the principle of parsimony) [68]. At the same time, when a complex model shows better predictive power, the complex model should be selected (section 2.8).

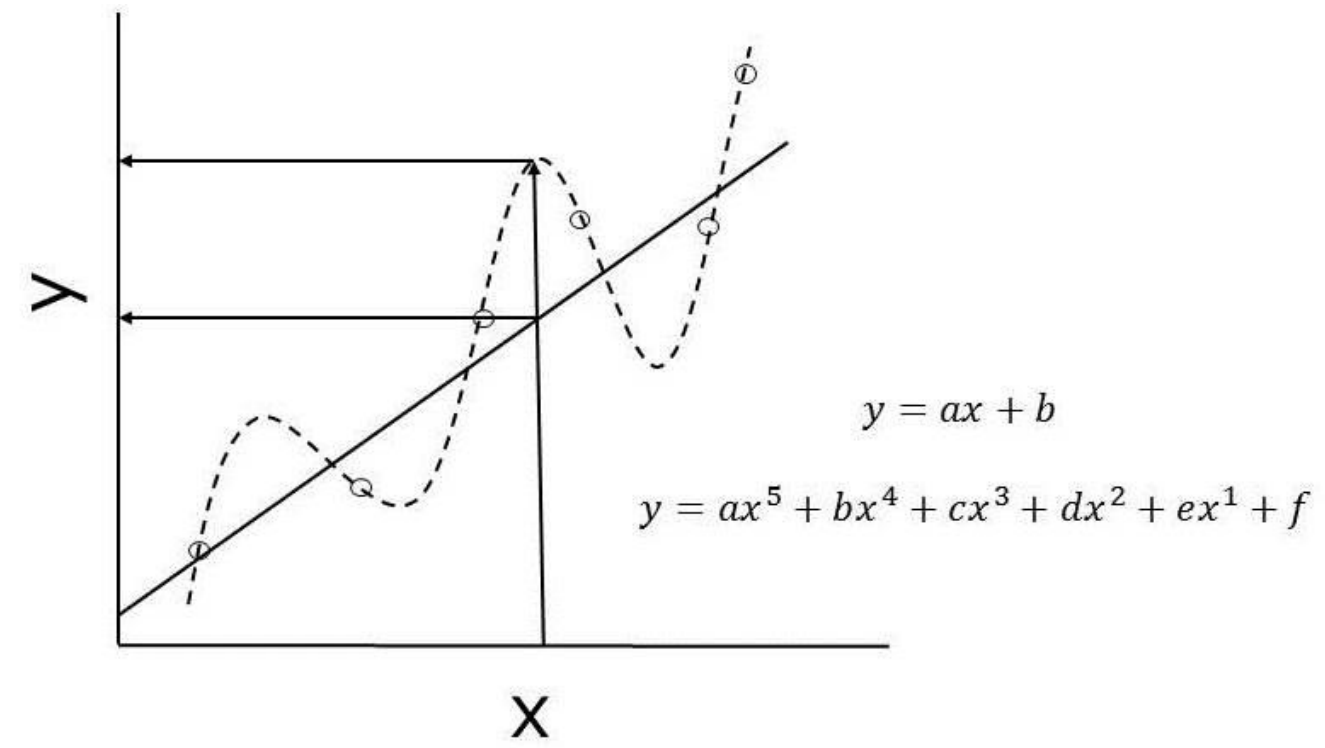

Figure 3. Overfitting.

In Figure 3, the quintic equation perfectly describes (fits) the data. If the experimental data is error-free, it is suitable for predicting unknown $Y$ from new $X$ data. In practice, especially for biological data, there is always error in experimental data. For objective model selection, statistical indices such as the Akaike Information Criterion (AIC) can be used [69].

\subsection{How to interpret a model}

There are several ways to interpret a mathematical model to understand the behavior of the system of interest.

\section{Parameters}

The first step to understanding a PBPK model is to know the parameters that affect the physical and physiological processes. Parameters that are not included in the model equation cannot be captured by the model. Before using a PBPK model, we must understand the key factors that affect the pharmacokinetic processes of a drug (sections 2.1 and 2.7). Intermediate parameters can be used to understand the contribution of each process. 


\section{Mathematical structure}

A mathematical structure represents the relationship between the system parameters, the dependent variables, and the independent variables, for example, being additive, synergistic, proportional, inversely proportional, exponential, etc. This point is important for parameter identifiability (see Part 3).

\section{Rate-limiting step}

In any kinetic model, it is essential to understand the rate-limiting step (or rate-limiting parameter) $[70,71]$. For example, the rate-limiting step that determines $F_{a}$ (FaRLS) can be diagnosed from the solubility, dissolution, and permeation numbers [53,72]. From FaRLS, the sensitive parameters for $F_{\mathrm{a}}$ of a drug can be easily identified. The rate-limiting step of intestinal membrane permeation can be diagnosed by using a physiologically-based permeability model $[73,74]$. The rate limiting step could differ depending on the physiology of each subject.

\section{Parameter sensitivity analysis}

Parameter sensitivity analysis (PSA) has been utilized to interpret black-box models, such as deep learning. PSA could also be useful in PBPK modelling. In PBPK modelling, PSA has been generally used to help deciding which parameters require further consideration either for additional in vitro measurement or parameter back-calculation (section 3.5). A PBPK model includes all parameters and models, however some of these may have little or no influence on the outcome.

\subsection{Evidence level regarding the predictive power of PBPK models}

The predictive power of a mathematical model can be evaluated based on the concept of evidencebased medicine (EBM) [15-17]. According to EBM, the evidence level of a case study is low. Case studies are prone to publication bias. Successful cases tended to be published whereas failed cases are usually not published. Historically, publication bias has caused the illusion of knowledge, especially in the medical area.

A systematic study is essential to evaluate the predictive power of PBPK models. For the bottom-up prediction by OA PBPK models, several systematic evaluation studies have already been published [2-5]. However, middle-out approaches (see Part 3 ) have been evaluated mostly by case studies (or the collection of case studies). Systematic evaluation is required for middle-out approaches using a standardized procedure (more precisely, for local middle-out dynamic PBPK models) $[10,75,76]$.

Although the evidence level of a case study is limited, it is still beneficial as far as appropriately conducted and reported. However, the creditability of case studies has often been compromised by the existence of a black box in a model (see Part 1) and inappropriate parameter optimization (see Part 3) [11]. The middle-out approach is discussed in detail in Part 3.

\subsection{Points to consider for systematic evaluation of predictive power}

Dataset: In OA PBPK modelling, the biopharmaceutical classification system (BCS) can be a good starting point $[6,77,78]$. A test set biased to BCS class I drug (high solubility/high permeability) should be avoided, because complete absorption is easily expected for BCS class I drug. Similarly, no food effect and no gastric $\mathrm{pH}$ effect on $F_{\mathrm{a}}$ are expected for BCS class I drugs (but they could affect $C_{\max }$ and $F$ ). In the case of BCS class I drugs, complex OA PBPK models may add little advantage over simple models for $F_{\mathrm{a}}$ prediction (depending on the purpose of the model) (section 2.8).

Control: A simple model or a naïve prediction (Figure 4) should be used as a control to evaluate the predictive power of complex models $[5,50,51,79]$. 
Outcome: The absolute bioavailability $(F)$ or $F_{\mathrm{a}}$ has been used to evaluate OA PBPK models [2]. $F$ is calculated by subtracting the effects of Dose and systemic clearance $(C L)$ from oral AUC data using i.v. data $\left(F=\left(\mathrm{AUC}_{\text {p.o. }} /\right.\right.$ Dose $\left._{\text {p.o. }}\right) /\left(\mathrm{AUC}_{\mathrm{i} . \mathrm{v} .} /\right.$ Dose $\left.\left._{\mathrm{i} . \mathrm{v} .}\right)\right) . F_{\mathrm{a}} F_{\mathrm{g}}$ can then be calculated from the hepatic clearance $\left(C L_{\mathrm{h}}\right)$ as $F_{\mathrm{a}} F_{\mathrm{g}}=$ $F / F_{\mathrm{h}}, F_{\mathrm{h}}=1-C L_{\mathrm{h}} /$ hepatic flow [80] $\left(F_{\mathrm{g}}\right.$ : the fraction escaping the intestinal wall metabolism, $F_{\mathrm{h}}$ : the fraction escaping the hepatic first-pass metabolism). In addition, there are several methods to estimate $F_{\mathrm{a}}$ from clinical PK data (section 2.6) $[5,81]$. AUC is less suitable for evaluating the predictive power of OA PBPK models because the main determinant of AUC is Dose and $C L$ in many cases.

Statistics: Statistics must be carefully interpreted. The percentage within a two-fold error is often used to evaluate the predictability of PBPK models. However, even when the predicted and observed $F$ values are randomly distributed from 0 to 1, the percentage within a two-fold error becomes $50 \%$ (Figure 4A) (see also Figures 3 and 4 in [6]). When predicted as average, $75 \%$ is within a 2 -fold error (Figure 4B). This kind of prediction is called "naïve prediction". This percentage increases when the data set is biased towards BCS class I.

(A) Random prediction

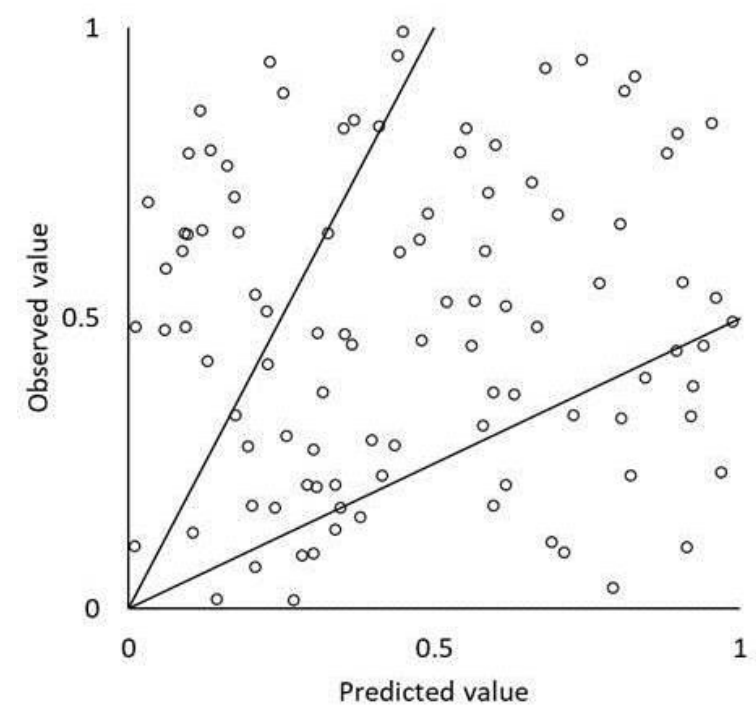

(B) Prediction as average

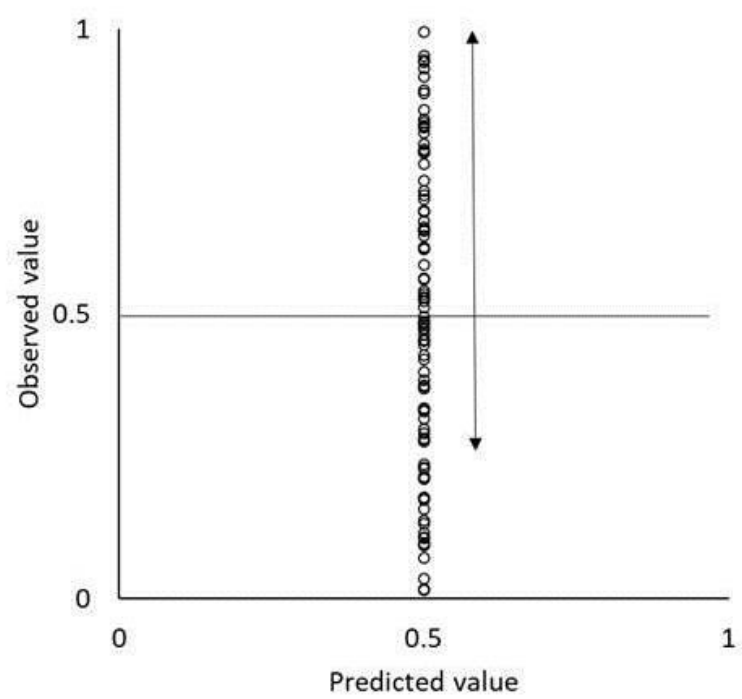

Figure 4. Naïve prediction. (A) Random prediction, (B) prediction by average. The percentage within a twofold error is $50 \%$ for (A) and $75 \%$ for (B).

\subsection{Surrogates of $F_{a}$ data}

In OA PBPK modelling, the fraction of a dose absorbed $\left(F_{\mathrm{a}}\right)$ is an important simulation output (section 1.3). However, there is no exact method to measure in vivo $F_{\mathrm{a}}$. Therefore, one or a few approximations have been used to estimate $F_{\mathrm{a}}$ from in vivo PK data $[50,70,79,82-84]$.

(A) Mass-balance data using a radio-labeled drug for i.v. and p.o.

(B) From absolute bioavailability $(F)$, hepatic clearance $\left(C L_{h}\right)$, and the hypothesis of $F_{\mathrm{g}}=1$ (section 3.8 (v)).

(C) Relative bioavailability of solution vs. solid formulation.

(D) Relative bioavailability in the fasted state vs. the fed state (when $D o<1$ in the fed state) (Do: the dose number (Dose/ $\left(S \times V_{\text {si }}\right)$ ), $S$ : solubility, $V_{\text {si: }}$ small intestinal fluid volume).

(E) Relative bioavailability between the dose strengths where $D o<1$ and $D o>1$ (AUC can be corrected by elimination $T_{1 / 2}$ for nonlinear clearance drugs).

The mass-balance data would be the most reliable data to estimate $F_{\mathrm{a}}$. However, these data are rarely available. When i.v. data is available, we can estimate $F_{a} F_{g}$ from $F$ and $C L_{h}$. When i.v. data are not available, 
for high permeability drugs, the relative bioavailability to an oral formulation that eliminates the effect of solubility and dissolution ((C)-(E)) can be used as a surrogate of $F_{\mathrm{a}}$ (cf. in this case, the effects of $F_{\mathrm{g}}$ and $F_{\mathrm{h}}$ are canceled out). The permeability category (low/high) can be reliably diagnosed by in vitro permeability assays [85]. In the case of high $P_{\text {app }}$ drugs ( $>$ metoprolol $P_{\text {app }}$ at $\mathrm{pH} 6.5$, metoprolol $\log D_{\text {pH6.5 }}=-1.1$ )[86], $F_{\mathrm{a}}$ will be greater than 0.8 with a very high probability when there is no solubility and dissolution limitation. Based on this high reliability, Caco-2 $P_{\text {app }}$ data has been used for regulatory biowaiver submission (note: for highly lipophilic drugs ( $\left.\log D_{\mathrm{pH} 6.5}>1.5\right)$, the Caco-2 assay may underestimate the permeability of the drug due to experimental artifacts such as membrane binding $[87,88]$, leading to a misassignment of a drug as low permeability (this can be identified by a mass balance study in Caco-2). In this lipophilicity range, the diffusion through the unstirred water layer becomes the rate-limiting step of in vivo membrane permeation. Therefore, a rough estimation of permeability from experimental log $D_{\text {pH6.5 }}$ will be sufficient and could be more reliable $[55,86])$.

The above methods showed similar $F_{\mathrm{a}}$ values when applied to the same drug in most cases $[50,79,82,83]$, confirming the validity of these methods. More than 600 clinical $F_{\mathrm{a}}$ data has been compiled from the literature and has been used to evaluate OA PBPK models $[5,50,73,79,83,86,89,90]$. For low $F_{\mathrm{a}}$ drugs the inter-subject variability is usually very high, and a clinical study may be an unrepresentative sample of the population.

\subsection{Often forgotten physicochemical mechanisms in OA PBPK modelling}

A good understanding of physicochemical processes in oral drug absorption is specifically important for OA PBPK modelling (see conclusion part). Below are some examples of physicochemical mechanisms that are often forgotten in OA PBPK modelling.

\section{Example 1: $P_{\text {eff }}$ estimation from in vitro data}

It is well known that the effective intestinal membrane permeability $\left(P_{\text {eff }}\right)$ is markedly affected by bile micelle binding [51,91-94], the unstirred water layer (UWL) [74,95-98], and the anatomical features (villi and fold structures) [99] (section 2.1) (cf. the $P_{\text {eff }}$ value includes the free fraction effects by definition in most cases of commercial software). However, these factors cannot be captured by an empirical equation of $P_{\text {eff }}=a P_{\text {app }}{ }^{b}\left(\log P_{\text {eff }}=A^{\prime}+B^{\prime} \log P_{\text {app }}\right)\left(P_{\text {app }}\right.$ : in vitro apparent permeability). This empirical equation cannot correctly explain and predict the food effect (especially the negative food effect) [51,91,100], the formulation effect (solubility-permeability trade-off)[101,102], and species differences [52,103]. The empirical coefficients, $a$ and $b$ (or $A^{\prime}$ and $B^{\prime}$ ), are usually determined by the regression analysis between $P_{\text {eff }}$ and $P_{\text {app }}$ using hydrophilic model drugs ( $\left.\log D<1\right)$ [31]. Therefore, lipophilic drugs are beyond the applicable range of the standard curve. In addition, $P_{\text {app }}$ is usually measured in the absence of bile micelles on the apical side and albumin on the basolateral side. The thick UWL in the in vitro systems mask the membrane permeability when not rigorously agitated [98,104]. Finally, and most importantly, this equation is an empirical correlation so that it should not be referred to as "physiologically-based". A mechanistic physiologically-based permeation model that considers these factors is available in the literature (section 2.1) [49-55] and in some commercial software products.

\section{Example 2: Dissolution in the stomach}

The gastric fluid is acidified by hydrochloric acid $(\mathrm{HCl})$. Because $\mathrm{HCl}$ is not an efficient buffer, the $\mathrm{pH}$ value of the gastric fluid increases when a free weak base drug is dissolved [105]. In addition, the solid surface $\mathrm{pH}$ is markedly increased by the dissolving free base molecules $[42,43,48,61,106,107]$. Therefore, the solid surface solubility ( $\left.S_{\text {surface }}\right)$ and the bulk phase solubility $\left(S_{\text {dissolv }}\right)$ must be differentiated in the 
Nernst-Noyes-Whitney equation (section 2.1) [35]. These two factors are important for predicting the effect of gastric $\mathrm{pH}$ on drug absorption. A mechanistic dissolution model that considers these factors is also available in the literature (section 2.1) $[42,43,48,61,79,106,107]$ and in some commercial software products.

\section{Example 3: Salt dissolution}

The dissolution modelling of a salt form drug is not that easy as one might imagine. The solid surface solubility of a salt is significantly higher than that of the free form ( $>100$-fold in most cases). However, the equilibrium solubility in the $\mathrm{pH}$-controlled region becomes the same regardless of the starting material is a free form or a salt form (unless the residual solid (equilibrium maker) show different solid forms) (cf. a salt form coverts to a free form in the $\mathrm{pH}$-controlled region). In drug discovery and development, the solubility of a drug substance is usually measured in well-buffered media after a sufficient incubation time to achieve equilibrium [108-111]. The small intestinal pH (about pH 6.5) is in the $\mathrm{pH}$-controlled region in most cases. In addition, a salt form may or may not show faster dissolution and more importantly supersaturation after dissolution, because the solid surface precipitation of a less soluble free form can inhibit the dissolution of its salt $[112,113]$. The mechanism of supersaturation and precipitation is not well understood at this moment. However, at least, it does not simply follow the first-order kinetics [114-119].

In addition, in silico models for the physicochemical properties of a drug are not so accurate as to be used for PBPK modelling [120]. Solubility measurements are not as easy as a modeler might imagine $[109,111,121]$. The $\mathrm{p} K_{\mathrm{a}}$ values change between $25^{\circ} \mathrm{C}$ and $37{ }^{\circ} \mathrm{C}[122,123]$. Enabling formulations such as amorphous solid dispersion [124], co-crystal [125,126], nanoparticles [127-129], and self-emulsifying drug delivery system $[130,131]$ also requires an in-depth understanding of physical chemistry for OA PBPK modelling. Further investigations on these points are required in the future (see conclusion part).

\subsection{A simple PBPK model or a complex PBPK model, which one to use?}

\section{"Everything should be made as simple as possible, but not simpler."}

Albert Einstein

As mentioned above, we are fully aware that there are various opinions among the modelers on the selection of mathematical models. A PBPK model should be selected considering the purpose of modelling and available data at each drug discovery and development stage. In the early drug discovery stages, a simple PBPK model may be sufficient. In the late drug development stages and after launch, a complex PBPK model may be required to investigate more complex clinical situations.

For the use in the early drug discovery stages, simple models can show sufficient prediction performance $[20,64,82,132,133]$. A simple OA PBPK model has shown good prediction performance for the fraction of a dose absorbed $\left(F_{\mathrm{a}}\right)[5,50,79,83,134]$ and plasma concentration $\left(C_{\mathrm{p}}\right)$ - time profiles [135-143]. For relative bioavailability $\left(F_{\text {rel }}\right)$ prediction, a simple OA-PBPK model is also available for the food effect (via bile micelles) and gastric acid effect predictions $[51,79,100]$. By multiplying AUC in the fasted state (or low gastric $\mathrm{pH}$ ) with $F_{\text {rel }}$ (i.e., AUC ratio), AUC in the fed state (or high gastric $\mathrm{pH}$ ) can be estimated. Minimal PBPK models have also been proposed to reduce the complexity of a model (see section 3.13 for metabolic DDI prediction) [21,144].

To explain and predict complex pharmacokinetics and population variation in late drug development and product life-cycle management, a PBPK model should have sufficient components. Commercial software products that implements a complex dynamic OA PBPK model would be especially useful for these purposes. 


\section{Part 3: Middle-out approach}

Part 2 discussed the interpretation and evaluation of the PBPK model. One of the most difficult parts of evaluating a PBPK model is the validity of the middle-out approach. Therefore, middle-out approaches are discussed in this part. Middle-out approaches have been widely utilized in real drug discovery and development [21]. They would be effective to improve various processes in drug development. At the same time, if they are inappropriately used, they could cause trouble. The purpose of this section is to discuss how to properly use the middle-out approach in OA PBPK modelling.

\subsection{The concept of the middle-out approach}

In a middle-out approach, some parameters of a PBPK model are derived from in vitro data (bottom-up), while others are derived from clinical PK data (top-down) on a drug-by-drug basis (see also section 3.14). In the following sections, this prediction scheme is referred to as a "local (drug-by-drug)" middle-out approach to differentiate it from a "global" middle-out approach (section 3.15) [145]. For drug-drug interaction (DDI) prediction, the local middle-out approach using a CYP specific inhibitor or substrate has been successfully used [21] (section 3.12). However, it has been pointed out that the inappropriate use of a local middle-out approach reduces the creditability of OA PBPK modelling $[17,20]$.

A middle-out approach brings an empirical model into PBPK modelling (Figure 5, the red line and square). Therefore, we should follow the good practice of empirical modelling: (i) Before back-calculation, parameter identifiability must be carefully examined (sections 1.4 and 3.2) [18-23,32,33]. (ii) The degree of freedom must be enough to avoid overfitting (section 2.2, Figure 3). (iii) The predictive power must be validated using clinical data that is not used to back-calculate the parameter (called "cross-validation") (section 3.11). (iv) The optimized empirical model should be used within the parameter space limited by the data used for back-calculation (so that as interpolation) (This is a general recommendation for empirical models. For the hybrid of empirical and mechanistic models, this point (iv) needs further in-depth discussion.).

\subsection{Parameter identifiability: a simple explanation}

The issue of parameter identifiability in biological mathematical modelling (including PBPK modelling) has been repeatedly warned in the literature [18-24]. However, this issue seems to have been overlooked in many case studies using a local middle-out approach. In this section, parameter identifiability is plainly explained focusing on OA PBPK modelling.

In a middle-out approach, the identifiability of a parameter(s) must be assessed before back calculating the parameter, whether the parameter(s) can be uniquely and reliably identifiable from the input-output data [18-24]. There are two types of parameter identifiability: statistical and structural. Statistical identifiability is related to the experimental error of the observed data. However, even with error-free data, a model parameter could be structurally non-identifiable.

To illustrate the concept of structural identifiability, let's consider an equation of $Y=a b X$, where $X$ is an explanatory variable, $Y$ is an object variable, and $a$ and $b$ are system parameters [23]. The lump quantity $a \cdot b$ is uniquely identifiable from $X$ and $Y$, while the individual parameters of $a$ and $b$ are non-identifiable (it is mathematically indefinite). Even when multiple $X Y$ data sets are available, $a$ and $b$ are non-identifiable. Therefore, $a$ or $b$ must be fixed separately. 
(A) Bottom-up approach
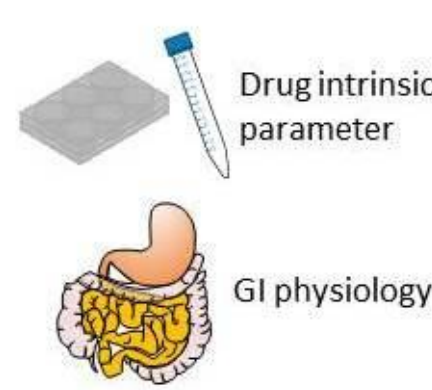
parameter GI physiology

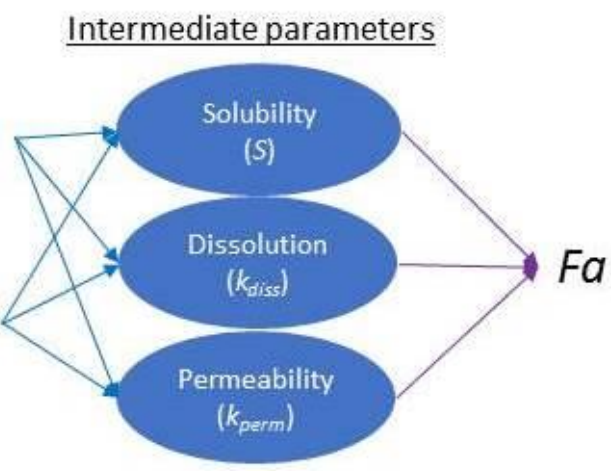

(B) Middle-out approach
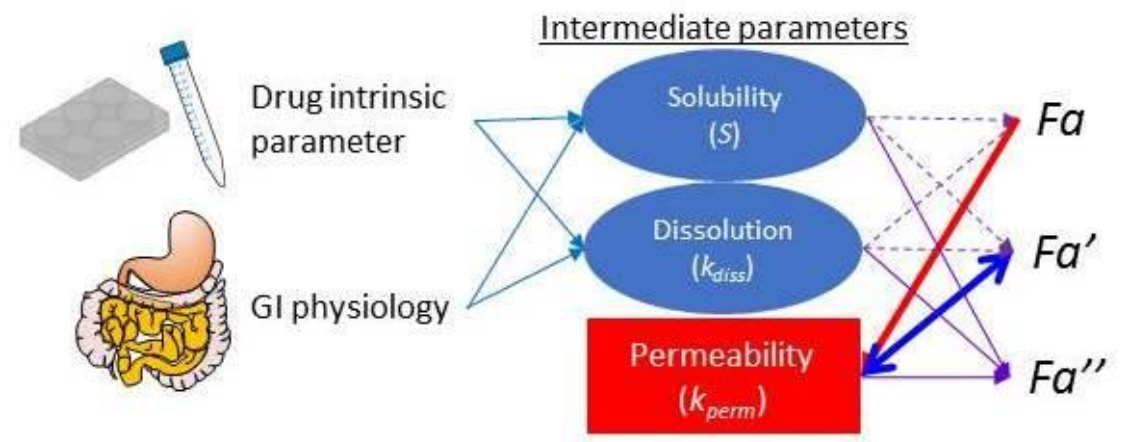

\section{Back calculate $k_{\text {perm }}$}

2. Validate $k_{\text {perm }}$

\section{Predict $F a^{\prime \prime}$}

Figure 5. Bottom-up and middle-out approaches for OA PBPK modelling. A middle-out approach brings an empirical model into a PBPK model (the red line and square). For parameter back calculation and validation, separate clinical data $\left(F_{\mathrm{a}}, F_{\mathrm{a}}{ }^{\prime}\right)$ must be used.

The statistical identifiability can be understood by considering $Y=1 /(X+a)$. If the error of $X$ is comparable to $a, a$ cannot be reliably determined (even assuming zero error in $Y$ ). For example, when $X=$ $100 \pm 10$, we cannot determine $a$ smaller than 10 . Preferably, $a$ should be determined at $X<<a$. Another familiar example is $Y=1-\exp (-a X)$ (Figure 6). In this case, $a X$ should be $<1.5$ if $Y$ has a $20 \%$ error. The other simple examples of parameter identifiability are shown in Supplemental Information.

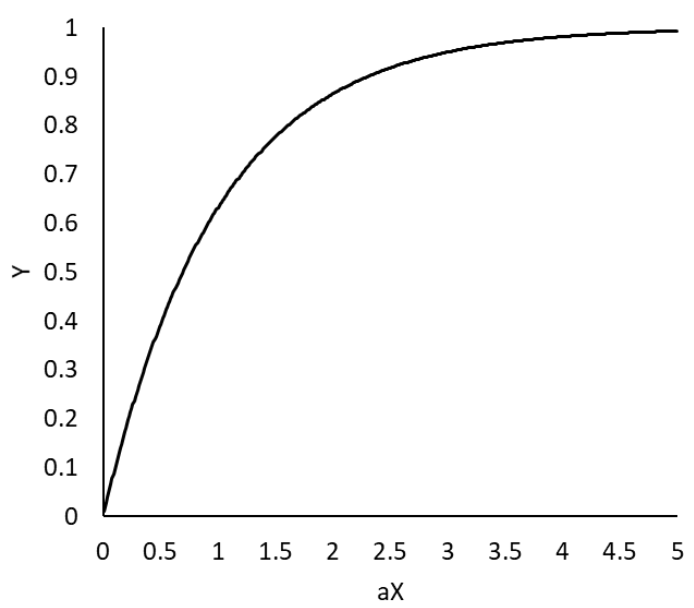

Figure 6. $Y=1-\exp (-a X)$.

\subsection{Local middle-out approach to predict the food effect: an example}

To overview the process of a local middle-out approach and understand the importance of parameter identifiability, in this section, we look at a simple example of OA PBPK modelling using a local middle-out approach and then discuss the checkpoints. 


\section{Step 1: Problem statement}

The drug candidate is a poorly soluble non-ionizable compound. The formulation is a simple immediaterelease formulation. Drug parameters are available from preclinical in vitro studies (Table 1). The physiological data are provided as default values.

Table 1. Drug and physiological parameters (example)

\begin{tabular}{lcl}
\hline Parameter & Value & Comments \\
\hline Drug parameter & & \\
Permeation rate constant $\left(k_{\text {perm }}\right)$ & $0.1 \mathrm{~h}^{-1}$ & From Caco-2 data $\left(P_{\text {eff }}=a P_{\text {app }}{ }^{b}\right)$ \\
Effective solubility $(S)$ & $0.1 \mathrm{mg} / \mathrm{mL}$ & Fasted state simulated intestinal fluid (FaSSIF) \\
& $0.2 \mathrm{mg} / \mathrm{mL}$ & Fed state simulated intestinal fluid (FeSSIF) \\
Dose strength (Dose) & $350 \mathrm{mg}$ & \\
Particle size & $5 \mu \mathrm{m}$ & \\
Diffusion coefficient & $6 \times 10^{-6}$ & \\
& $\mathrm{~cm} / \mathrm{s}$ & \\
& & \\
Physiological parameter & & \\
Small intestinal fluid volume $\left(V_{\text {si }}\right)$ & $1000 \mathrm{~mL}$ & Default (see Checkpoint 4) \\
Small intestinal transit time $\left(T_{\text {si }}\right)$ & $3.5 \mathrm{~h}$ & Default \\
Small intestinal radius $\left(R_{\text {si }}\right)$ & $1.5 \mathrm{~cm}$ & Default \\
Degree of flatness $(D F)$ & 1.7 & Default \\
Fraction escaping first pass hepatic & 1 & Calculated from i.v. $C L_{\mathrm{h}}$ and hepatic flow \\
metabolism $\left(F_{\mathrm{h}}\right)$ & & \\
\hline
\end{tabular}

Now, the clinical PK data in the fasted state became available after the first-in-human study (both p.o. and i.v.). The bioavailability $(F)$ was $0.30 \pm 0.15$ (mean \pm s.d.). We are asked by the managers to predict AUC in the fed state in healthy volunteers. To utilize the first-in-human clinical data, we decided to conduct a local middle-out approach.

Step 2: Model selection

The particle size is small enough so that the drug dissolution process does not become the rate-limiting step [70-72]. In addition, because the drug is non-ionizable, gastric dissolution would have little effect on $F$. In the case of solubility limited oral absorption, $F$ can be calculated as [146]:

$$
F=F_{\mathrm{a}} F_{\mathrm{g}} F_{\mathrm{h}}=F_{\mathrm{g}} F_{\mathrm{h}} \frac{P n}{D o}=\frac{F_{\mathrm{g}} F_{\mathrm{h}} k_{\text {perm }} S V_{\mathrm{si}} T_{\mathrm{si}}}{D o s e}
$$

where $P \mathrm{n}$ is the permeation number $\left(=k_{\text {perm }} \times T_{\text {si }}\right)$, and $D o$ is the dose number Do $=D o s e /\left(S \times V_{\text {si }}\right) . F_{\mathrm{g}}$ is the fraction escaping the intestinal metabolism. In this example, Eq. 1 is used as a model equation for convenience.

Step 3: Parameter optimization

From Eq. 1 and given data, $k_{\text {perm }}$ is optimized to be $0.3 \mathrm{~h}^{-1}$ in the fasted state.

Step 4: Model validation

Using the optimized $k_{\text {perm }}$ value, $F$ is calculated as,

$$
F=\frac{0.3 \times 0.1 \times 1000 \times 3.5}{350}=0.30
$$

This predicted $F$ value perfectly matches the clinical $F$ data $(=0.30)$. This $k_{\text {perm }}$ value is additionally 
validated using the independent clinical $F$ data in the fasted state.

\section{Step 5: Prediction}

The optimized $k_{\text {perm }}$ value is then used to predict $F$ in the fed state. Using the solubility in FeSSIF $(0.2$ $\mathrm{mg} / \mathrm{mL}$ ), $F_{\mathrm{a}}$ is calculated as

$$
F=\frac{0.3 \times 0.2 \times 1000 \times 3.5}{350}=0.60
$$

Therefore, AUC is predicted to increase twofold in the fed state.

\section{Checkpoint 1: Hidden "bottom-up" prediction}

Usually, we start a prediction study with a "bottom-up" approach at a preclinical stage (section 1.3). The input parameters are initially projected from the in vitro data. In the above case,

$$
F=\frac{0.1 \times 0.1 \times 1000 \times 3.5}{350}=0.10
$$

The "bottom-up" prediction resulted in a 3-fold underestimation. $k_{\text {perm }}$ was increased threefold (from 0.1 to 0.3 ) after parameter optimization. This is not a subtle adjustment. This information alerts that there is a marked discrepancy between the $k_{\text {perm }}$ values estimated from the Caco- 2 data and back-calculated from the clinical $F$ data (section 3.4).

\section{Checkpoint 2: Subject of parameter optimization}

The reason for selecting $k_{\text {perm }}$ as the subject of parameter optimization is not clear (sections 1.2 to 1.4 and 3.5 to 3.7$)$.

\section{Checkpoint 3: Parameter identifiability}

Eq. 1 suggests that the $k_{\text {perm }}$ value is not identifiable from the provided data $\left(F, F_{\mathrm{h}}\right.$, Dose, $S, V_{\mathrm{si}}$, and $\left.T_{\mathrm{si}}\right)$. $F_{\mathrm{g}}$ must be separately fixed (section $3.8(\mathrm{v})$ ). In the above calculation, $F_{\mathrm{g}}$ was unknowingly assumed to be 1 (section 1.2 to 1.4 ).

\section{Checkpoint 4: Hidden errors}

The intestinal fluid volume of $1000 \mathrm{~mL}$ is significantly greater than the current best estimate (most probably $<150 \mathrm{~mL}$ ) (in the discussion below, we assume that $100 \mathrm{~mL}$ is realistic for the convenience of discussion) [26-29]. The optimized $k_{\text {perm }}$ value carries the error in the intestinal fluid volume (e.g., $1000 \mathrm{~mL}$ vs $100 \mathrm{~mL}$ ). With a more realistic intestinal fluid volume of $100 \mathrm{~mL}$, the $k_{\text {perm }}$ value is back-calculated to be $3.0 \mathrm{~h}^{-1}$, ten-fold higher than $0.3 \mathrm{~h}^{-1}$. This could cause a misunderstanding of the rate-limiting step for membrane permeation (section 1.4).

\section{Checkpoint 5: Variation of clinical data}

Considering that $\mathrm{CV} \%$ of $F$ is $50 \%$, it is not possible to back-calculate the $k_{\text {perm }}$ value within a good confidence interval.

\section{Checkpoint 6: Constancy of optimized parameter}

It was implicitly assumed that the $k_{\text {perm }}$ values in the fasted and fed states are the same. However, it is well known that bile micelle binding reduces the free fraction, the effective diffusion coefficient, and consequently $P_{\text {eff }}$ and $k_{\text {perm }}$ (section 2.7$)$ [51,91,92,94,147,148]. 


\section{Checkpoint 7: Model validation}

The predictive power of a model cannot be validated by the same data used for parameter backcalculation (section 3.11).

\section{Checkpoint 8: Prediction}

After parameter optimization, the predictive range of the optimized model is limited to the parameter space of the clinical data that is used for model development (limited to interpolation with some exceptions).

In the above example, a simple model (Eq. 1) was used for convenience to illustrate the middle-out approach. In complicated PBPK modelling, these checkpoints are not so easy to recognize. However, the mathematical principle remains the same.

\subsection{When is a local middle-out approach required?}

A local middle-out approach is required when a bottom-up prediction is not satisfactory for the contest of use (section 1.3, Figure 1). The widespread use of a local middle-out approach is consistent with the results of systematic evaluation suggesting that current bottom-up OA PBPK models need significant improvement (introduction section). Discrepancies between bottom-up predictions and clinical observations may suggest opportunities to uncover unidentified mechanisms or to improve the equations and parameters of the model (Sections 1.3, 3.3 Checkpoint 4). Before parameter back-calculation, the reason for the discrepancy should be thoroughly explored (sections 1.4 and 2.7).

\subsection{How to diagnose parameter identifiability in OA PBPK modelling}

In the literature, $P_{\text {eff }}$ has often been the subject of back-calculation. Therefore, the identifiability of $P_{\text {eff }}$ is discussed as an example below.

Simple analytical solutions are useful for diagnosing the parameter identifiability (section 2.2). The $F_{\mathrm{a}}$ equation represents the relationship between the solubility, dissolution rate, and permeation of a drug to $F_{\mathrm{a}}[5,35,149]$ :

$$
F_{\mathrm{a}}=1-\exp \left(-\frac{1}{\frac{1}{D n}+\frac{D o}{P n}}\right)
$$

where $D \mathrm{n}$ is the product of the dissolution rate $\left(k_{\text {diss }}\right)$ and the intestinal transit time $\left(D n=k_{\text {diss }} \times T_{\text {si }}\right)$. When $D o<1$, set $D o=1$.

When the oral absorption of a drug is limited by the dissolution rate $(D n<P n / D o)$, the permeation process is statistically non-identifiable from $F_{\mathrm{a}}$ (cf. $Y=1 /(X+a)$ ). In other words, for $P_{\text {eff }}$ to be identifiable, the oral absorption must be permeability or solubility-permeability limited. In this case, Eq. 2 becomes:

$$
F_{\mathrm{a}}=1-\exp \left(-\frac{P n}{D o}\right)
$$

At $P \mathrm{n} / D \mathrm{Do}>0.7$, the $P_{\text {eff }}$ value (that is in $P \mathrm{n}$ ) is statistically non-identifiable from clinical $F_{\mathrm{a}}$ data considering the variation of clinical $F_{\mathrm{a}}$ data (cf. $Y=1-\exp (-a X)$, Figure 6). At $P \mathrm{n} / D \mathrm{o}<0.7$, Eq. 3 can be approximated to [146]: 


$$
F_{\mathrm{a}} \approx \frac{P n}{D o}
$$

Using Eq. 4 for $F_{\mathrm{a}}$, the AUC value after oral administration can be expressed as:

$$
A U C=\frac{\text { Dose }}{C L} F=\frac{\text { Dose }}{C L} F_{\mathrm{a}} F_{\mathrm{g}} F_{\mathrm{h}}=\frac{\text { Dose }}{C L} \frac{P n}{D o} F_{\mathrm{g}} F_{\mathrm{h}}=\frac{\text { Dose }}{C L} \frac{k_{\text {perm }} T_{\mathrm{si}}}{\frac{\text { Dose }}{S V_{\mathrm{si}}}} F_{\mathrm{g}} F_{\mathrm{h}}=\frac{F_{\mathrm{g}} F_{\mathrm{h}} k_{\mathrm{perm}} S V_{\mathrm{si}} T_{\mathrm{si}}}{C L}
$$

Finally,

$$
A U C=A U C=\frac{F_{\mathrm{g}} F_{\mathrm{h}} 2 \cdot D F \cdot P_{\mathrm{eff}} S V_{\mathrm{si}} T_{\mathrm{si}}}{C L R_{\mathrm{si}}}
$$

In the absence of a unique correspondence between a parameter and a $C_{p}$ - time profile, it is impossible to directly quantify the physiological process that involves the parameter. When the parameters are in a multiplication relationship, they are structurally non-identifiable from clinically observed data (cf. $Y=a b X$ ). As clearly represented in Eq. 6, the individual parameters related to oral drug absorption cannot be calculated solely using AUC after oral administration [19-22,33]. Therefore, an OA PBPK model is essentially overparameterized. To back-calculate $P_{\text {eff }}$ from $F_{\mathrm{a}}$, the other eight parameters $\left(F_{\mathrm{g}}, F_{\mathrm{h}}, S, V_{\mathrm{si}}, T_{\mathrm{si}}, R_{\mathrm{si}}, D F\right.$, and $C L$ ) must be fixed separately.

Parameter sensitivity analysis can be used as a support to diagnose the identifiability of parameters. However, AUC is sensitive to all the parameters on the right-hand side of Eq. 6. Being a sensitive parameter is a necessary but not sufficient condition to be identifiable [19-22].

A similar analysis of parameter identifiability can be performed for $C_{\max }$ and $T_{\max }$ :

$$
\begin{aligned}
& C_{\max }=\frac{F D o s e}{V_{\mathrm{d}}}\left(\frac{k_{\mathrm{a}}}{k_{\mathrm{el}}}\right)^{\frac{k_{\mathrm{el}}}{k_{\mathrm{el}}-k_{\mathrm{a}}}} \\
& T_{\max }=\frac{1}{k_{\mathrm{a}}-k_{\mathrm{el}}} \ln \left(\frac{k_{\mathrm{a}}}{k_{\mathrm{el}}}\right)
\end{aligned}
$$

where $k_{\mathrm{a}}$ is the absorption rate constant, $k_{\mathrm{el}}$ is the elimination rate constant, and $V_{\mathrm{d}}$ is the volume of distribution. $k_{\mathrm{a}}$ can be approximated as $1 / k_{\mathrm{a}}=1 / k_{\text {diss }}+D o / k_{\text {perm }}$ (section 3.14 ).

\subsection{What kind of parameters can be candidates for back-calculation?}

Only an intermediate parameter (e.g., $P_{\text {eff }}$ ) or an empirical scaling factor should be the candidates for the subject of parameter back-calculation from clinical PK data in a local middle-out approach (Figure 2).

Drug-intrinsic parameters, especially physicochemical properties, should not be the subject of parameter back-calculation from clinical PK data. They should be determined by in vitro measurements.

Physiological parameters such as the small intestinal fluid volume $\left(V_{s i}\right)$ should not be the subject of drugby-drug back-calculation as well, because they do not change drug-by-drug (except for drugs with gastrointestinal effects) (A global middle-out approach has been used to estimate some physiological parameters when direct measurements are not possible (section 3-15)).

Post-absorptive (systemic) PK parameters such as $C L$ and $V_{d}$ should be determined from i.v. $C_{p}$ - time data [33] (unless $F=1$ can be surely estimated from in vitro data, such as the case of BCS class I drug with low hepatic clearance). The same $C L$ and $V_{d}$ values should be used regardless of formulations or 
administration routes. Oral formulation usually does not affect systemic $C L$ and $V_{d}$ (however, it affects $C L / F$ and $\left.V_{\mathrm{d}} / \mathrm{F}\right)$.

\subsection{Which candidate parameter should be selected for back-calculation?}

When multiple candidate parameters can equally explain the discrepancy between prediction and observation, we cannot determine which one to be selected for back-calculation solely based on the clinical data. This situation is like a checksum process. We can validate a series of numbers by checking the sum of numbers, but we cannot tell which number has the error. The selection of a parameter depends on the clinical study design and the reliability of the estimation from in vitro data (section 3-8, (iv)). Identifiability issues can be even more severe when there are regional differences in the gastrointestinal parameters in Eq 6.

\subsection{How to fix the other parameters}

There are several methods to fix the other parameter values.

\section{(i) Clinical study using different administration routes and formulations}

To reliably estimate a parameter from clinical PK data, the interference of confounding factors must be reduced as much as possible. A specific clinical study design has been employed to eliminate the confounding factors for each parameter (Table 2) (see also (3.13)). For example, systemic clearance (CL) can be obtained from i.v. data. An i.v. administration eliminates the oral absorption process $(F=1)$, so that $C L$ become identifiable from AUC (cf. $C L=$ Dose/AUC). $V_{\mathrm{d}}$ and $F_{\mathrm{h}}$ can also be calculated from the i.v. data [150]. An oral solution formulation can be used to eliminate $D n$ from Eq. 2 (1/Dn becomes negligible), and $S$ and $V_{\text {si }}$ from Eq. 6 (mathematically, by fixing $D o=1$ ).

Table 2. The parameters and the clinical study design

\begin{tabular}{lll}
\hline Parameter & Clinical study design & Reference \\
\hline$C L, V_{\mathrm{d}}$ & i.v. administration & a $^{\mathrm{a}}$ \\
$P_{\text {eff }}$ & Site-specific solution administration and i.v. administration & {$[151]$} \\
Precipitation rate & Site-specific solution administration and sampling & {$[152][153]$} \\
\hline${ }^{\mathrm{a}}$ See any pharmacokinetic textbook &
\end{tabular}

It is not known whether parameter back-calculation from the oral $C_{p}$ - time data after the administration of solid dosage forms can be accurate. Theoretically, the dissolution processes can be decomposed from the permeation process by using the $C_{p}$ - time data from solution formulations [154]. However, this deconvolution process often becomes unstable due to the variation of the data. In addition, there has been no systematic evaluation of $P_{\text {eff }}$ back-calculation from the oral $C_{p}$ - time data after the administration of solid dosage forms. Unfortunately, in the literature, $P_{\text {eff }}$ has been back-calculated even in the absence of i.v. data in many cases. The credibility of such $P_{\text {eff }}$ back-calculation is at least questionable (section 3.9).

\section{(ii) Experimentally measured physiological parameters}

Experimentally measured physiological parameters can be obtained from the literature $\left(V_{\mathrm{si}}, T_{\mathrm{si}}\right.$, and $R_{\mathrm{si}}$ in Eq. 6). However, it should be noted that some of the physiological parameters reported in the literature have large variations between subjects and between occasions.

(iii) Physiological parameters estimated by a global middle-out approach

The global middle-out approach has been used to back-estimate an unknown physiological parameter from clinical PK data ( $D F$ in Eq. 6, section 3.15). 


\section{(iv) Preclinical in silico, in vitro, and in vivo data}

Preclinical in silico, in vitro, and in vivo data can be used to fix a parameter if their predictability is sufficient considering the contest of use (COU) (section 1.2). The in vitro equilibrium solubility in biorelevant media such as FaSSIF and FeSSIF has often been assumed to be in vivo predictive. However, it should be noted that these are simplified artificial fluids, not actual intestinal fluids. FaSSIF and FeSSIF are very good model fluids, yet, the solubility values do not always accurately reflect the real in vivo values [155]. In addition, the solubility data could be inaccurate when inappropriately measured [109].

Current in vitro-in vivo extrapolation (IVIVE) and allometric scaling for $C L$ and $V_{d}$ is not sufficiently accurate for the COUs of OA PBPK modelling in the late drug development stage [7].

In silico models for the physicochemical properties and other ADME properties of a drug are not so accurate as to be used for PBPK modelling [120] (except for molecular diffusion coefficients [156, 157]).

\section{(v) Hypothesis generation}

The $P_{\text {eff }}$ value is still not identifiable after fixing $S, V_{\mathrm{s}}, T_{\mathrm{si}}, R_{\mathrm{si}}, D F, C L$, and $F_{\mathrm{h}}$. Hypothesis generation is required to estimate $F_{\mathrm{g}}$ [14]. Hypothesis generation may include IVIVE, but in a less qualitative manner. Hypothesis generation about the negligibility of a parameter would be most credible and useful because it can reduce the interference from the parameter for back-calculation. The credibility of a hypothesis can be improved by combining various in vitro, preclinical in vivo, and clinical observations. For example, when in vitro data suggest that a drug is not a substrate of intestinal metabolic enzymes, $F_{\mathrm{g}}=1$ (no gut wall metabolism) can be a plausible hypothesis. Low intrinsic hepatic clearance $(<100 \mathrm{~mL} / \mathrm{min} / \mathrm{kg})$ [158], the lack of metabolites, and the lack of clinical grapefruit-drug interaction can further support the hypothesis of $F_{\mathrm{g}}$ $=1$.

\subsection{A good example of credible parameter back-calculation}

Sjogren et al. reported that $P_{\text {eff }}$ can be credibly identifiable by the deconvolution of the $C_{p}$ - time profiles after intraintestinal bolus administration as a solution, using i.v. disposition data [151]. They pointed out that the $C_{\mathrm{p}}$ - time profiles after an oral administration may not be suitable for $P_{\text {eff }}$ estimation due to possible interference of confounding factors. They used a solution formulation to eliminate the uncertainty in $S, V_{\text {si, }}$ and the effect of dissolution processes. Intra-intestinal administration was used to eliminate the effect of gastric emptying. The i.v data was used to calculate $C L$ and $F_{\mathrm{h}}$. Hypothesis generation was used for $F_{\mathrm{g}}$. The basic concept of their approach is shown in Figure 8 (They used the deconvolution method, but it was simplified to AUC calculation to explain the concept).

\subsection{Accumulation of errors}

After parameter back-calculation, the simulation curve would show perfect fitting to the observed $C_{p}$ time data that had been used for parameter back-calculation. However, this perfect fitting does not imply the validity of all parameters and model equations.

A back-calculated parameter inherits the errors of the other parameters (see Checkpoint 4). In the case of Eq. 6, the errors in nine separately fixed parameters $\left(V_{\mathrm{si}}, T_{\mathrm{si}}, R_{\mathrm{si}}, D F, S, C L, F_{\mathrm{g}}, F_{\mathrm{h}}\right.$, and clinical AUC) exponentially accumulate in $P_{\text {eff }}$ (sections $2.2,2.8$ ). Even if each parameter has only a small error of $20 \%$ (1.2-fold), the total error can become 5.2 -fold $\left(=1.2^{9}\right)$. To back-calculate $P_{\text {eff }}$ within less than $20 \%$ error, each parameter must have less than $2 \%$ error. Any experimentalist knows that this is not possible. Furthermore, back-calculating one parameter hides the errors of the other parameters and model equations (section 3.4). $P_{\text {eff }}$ itself can show high inter-subject variability [159]. 


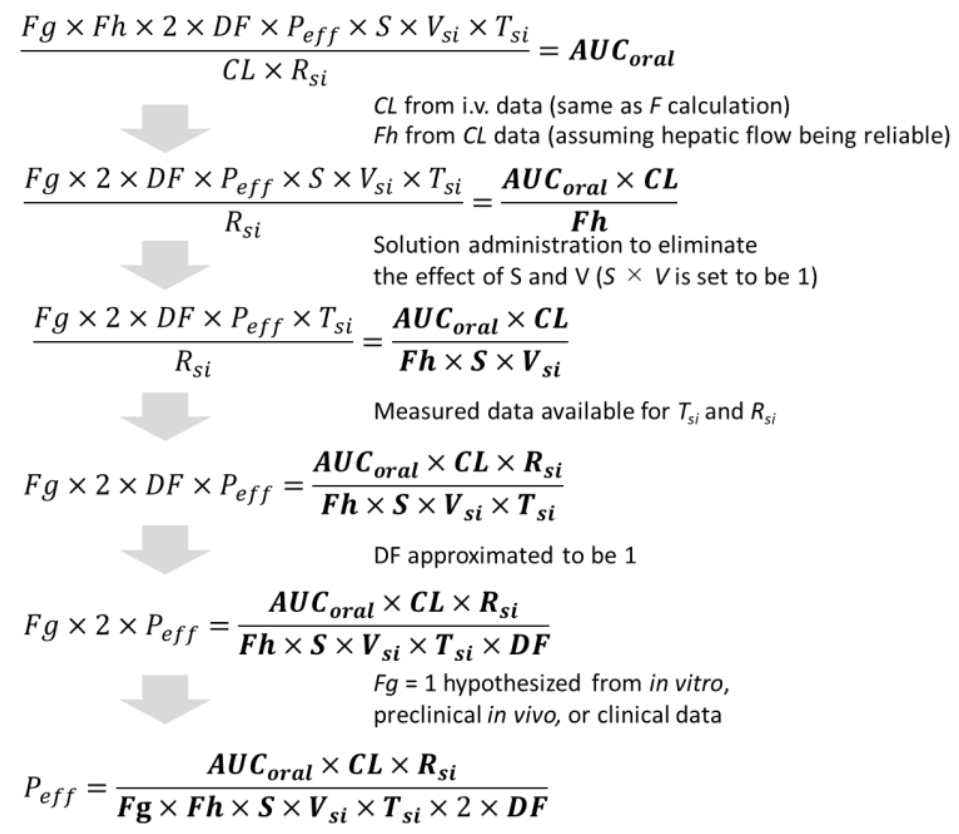

Figure 8. $P_{\text {eff }}$ calculation scheme using a set of clinical data, literature data, and hypothesis generation. Each parameter is fixed step-by-step. The parameters after the evaluation are moved to the right side of the equation and shown in bold type. Eq. 6 is used to understand the concept of back-calculation. Sjögren et al. used the deconvolution method [151], but the basic concept of parameter identification is the same (the deconvolution method can eliminate the uncertainty in $T_{\mathrm{si}}$ ).

\subsection{How to validate the predictive power of a PBPK model after parameter back-calculation}

In a local middle-out approach, the predictive power needs to be validated for each drug after parameter back-calculation. The parameter back-calculated in one clinical condition is not necessarily valid in the other clinical condition (section 3.3, checkpoint 6). The predictive power of a model cannot be validated by the same data used for parameter back-calculation, because it is self-referencing. Therefore, the predictive power needs to be validated using other clinical PK studies of the context of use (COU) ("cross-validation"). The PK data under clinical conditions where some of the same pathways in the system are perturbed as in the COU can be employed for validation $[10,21]$. In the case of food effect prediction, the optimized model should be validated by the $C_{p}$ - time data under a clinical condition in which the pathways of oral absorption are perturbed by the same factor for the food effect. In other words, the food effect prediction must be validated by a clinical food effect study.

The clinical PK data in the same clinical condition cannot be used for validation, even if it is independently determined, because the $C_{p}$ - time profiles are expected to be similar under the same clinical condition (this is a kind of "leakage" in the cross-validation process). Multiple-dose PK data under the same clinical condition cannot be used for validation (unless the context of use is the prediction of metabolic enzyme induction or mechanism-based inhibition in the intestine after multiple-dose PK).

\subsection{2. "Confirm and refine" strategy in drug discovery and development}

The quality and quantity of experimental data available for PBPK modelling increase as a research project proceeds in drug discovery and development. Therefore, it would be beneficial to utilize these data for PBPK modelling (section 2.8).

Each module in an OA PBPK model can be confirmed by comparison with corresponding in vitro experimental data covering a wider range than in vivo conditions. For example, the solubility model should be validated by an experimental $\mathrm{pH}$-solubility profile in the range of $\mathrm{pH} 1.0$ to 8.0 to cover in vivo gastrointestinal conditions. The dissolution model (the Nernst-Noyes-Whitney equation) can be confirmed 
by comparison with dissolution test data. If the prediction result deviated from the experimental observation, an empirical correction parameter can be introduced, like the $z$ factor for each dissolution condition [81].

Similarly, in vivo animal PK data can be used to inform the confidence level of a PBPK model. However, in this case, the refinement (back-calculation) process requires the same cautions as the local middle-out approach. In addition, there may be species differences in the back-calculated parameter.

First-in-human PK data can be used to inform the confidence level of a PBPK model in humans. The PK data at a low dose (dose number $\ll 1$ ) can be used to evaluate the oral absorption process without interference from the dissolution rate and solubility. In the case of high permeability drugs, the low dose PK data may provide information regarding systemic clearance and volume of distribution to some extent (but i.v. PK data is preferable). The confidence level of solubility and permeability values (as Pn/Do, Eq. 2) may also be evaluated by the dose sub-proportionality of AUC (so that relative bioavailability between low and high doses). However, as discussed above, it is not easy to accurately back-calculate (refine) a parameter from in vivo oral PK data. Extreme caution should be exercised when using a middle-out approach.

\subsection{What is the difference between metabolic DDI and food effect predictions?}

A local middle-out approach has been successfully used to predict metabolic DDI by PBPK modelling $[12,160,161]$. Because it is practically impossible to clinically evaluate all DDI combinations, DDI prediction by a local middle-out approach would be of great value. In the local middle-out approach for DDI, to identify the fraction of metabolic clearance $\left(f_{m}\right)$ (e.g., a CYP isozyme), a specific inhibitor has been used in a clinical PK study (section 3.8 (i)). In the following, the concept of DDI prediction by the local middle-out approach is briefly explained (see $[12,160,161]$ for details).

In the case of i.v. dosing (or $F=1$ for oral dosing), $C L$ and AUC in the absence of an inhibitor can be expressed as:

$$
\begin{aligned}
& C L=C L\left(f_{\mathrm{mA}}+F_{\mathrm{mB}}\right), \quad F_{\mathrm{mA}}+F_{\mathrm{mB}}=1 \\
& \mathrm{AUC}=\frac{\text { Dose }}{C L\left(f_{\mathrm{mA}}+f_{\mathrm{mB}}\right)}
\end{aligned}
$$

where $f_{\mathrm{mA}}$ and $f_{\mathrm{mB}}$ are the fractions of metabolic clearance pathways ( $\mathrm{A}$ and $\mathrm{B}$, respectively). The $f_{\mathrm{mB}}$ can be calculated from the $A \cup C$ ratio in the absence $\left(A \cup C_{n o-i n h i b i t i o n}\right)$ and presence $\left(A \cup C_{\text {inhibition, }}, f_{m B}=0\right)$ of a specific strong inhibitor as:

$$
f_{\mathrm{mB}}=1-\frac{\mathrm{A} U \mathrm{C}_{\text {no-inhibition }}}{\mathrm{AUC} \mathrm{C}_{\text {inhibition }}}
$$

The inhibitor should specifically inhibit only pathway B and there is no other underlying clearance mechanism (e.g. renal clearance) or uptake/efflux transporter in interplay which can be influenced by the inhibitor. In this case, the $f_{\mathrm{mB}}$ value is identifiable from the AUC ratio. This equation also suggests that the AUC ratio is predictable without using any complex PBPK modelling. A simple prediction scheme for metabolic DDI (AUC ratio) has been proposed and thoroughly validated using a large number of clinical DDI data (section 2.8) [67,162-164].

However, a similar approach cannot be simply applied to the food effect prediction, because a food intake simultaneously affects various processes of oral drug absorption (section 2.7) [165].

\subsection{What is the difference between PBPK and compartmental PK models?}


In pharmacokinetics, the compartmental PK model is widely used. Although both compartmental PK models and PBPK models belong to mathematical models, their applications are different.

In compartmental PK models, all parameters are calculated from clinical PK data ("top-down" approach) (section 3.1). Because the compartmental PK model is empirical, it is used following the good practice of empirical modelling (section 3.1). To avoid overfitting (section 2.2, Figure 3), the Akaike information criterion has been used to select the number of compartments appropriately [69]. For an i.v. PK model, the number of compartments is set to be one or two in most cases ( 2 and 4 parameters, respectively). For an oral PK model, only three parameters, that is, $k_{\mathrm{e}}, k_{\mathrm{a}}$, and $V_{\mathrm{d}} / \mathrm{F}$ are used in most cases (Figure 7).

$$
\begin{cases}C p(t)=\frac{\text { DoseFgFh }}{V d} \frac{k_{a}}{k_{a}-k_{e l}}\left(\exp \left(-k_{e l} t\right)-\exp \left(-k_{a} t\right)\right) & t<T \\ C p(t)=C p(T) \exp \left(-k_{e l}(t-T)\right) & t>T\end{cases}
$$

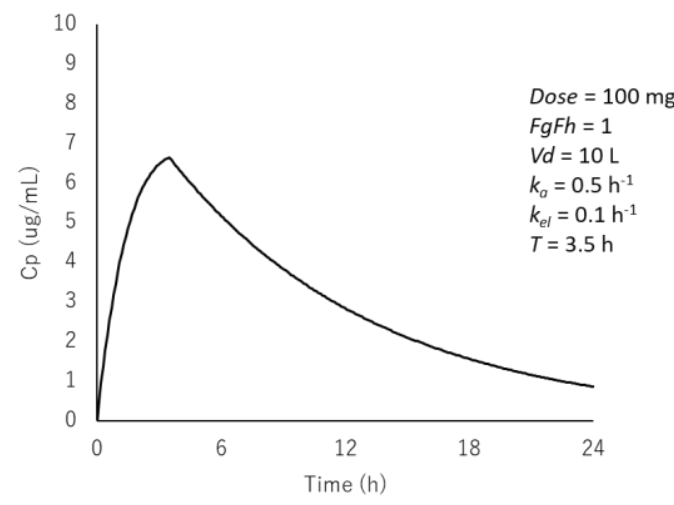

Figure 7. An oral one-compartment model with an finite absorption time of $T=3.5 \mathrm{~h}[86,166]$. In many cases, an oral $C_{p}$ - time profile can be summarized into three parameters, $k_{a}$, the elimination rate constant $\left(k_{\mathrm{el}}\right)$, and the lumped values of the volume of distribution $\left(V_{\mathrm{d}}\right), F_{\mathrm{g}}$, and $F_{\mathrm{h}}\left(V_{\mathrm{d}} / F_{\mathrm{g}} F_{\mathrm{h}}\right) . T_{\max }, C_{\max }$ and AUC are all described by using only one absorption parameter $\left(k_{\mathrm{a}}\right)$ that represents the oral absorption of a drug. Therefore, the oral absorption of a drug can be well described by fitting one of many conjugated parameters in a complex OA PBPK model (the degree of freedom is zero). From the $C_{\mathrm{p}}$-time profile after oral administration, only the composite parameter of $V_{\mathrm{d}} / F_{\mathrm{g}} F_{\mathrm{h}}$ is identifiable. An i.v. data is required to fix $V_{\mathrm{d}}$ (and CL) [33].

In contrast, a PBPK model consists of dozens to hundreds of parameters (section 2.1) [37]. All these parameters cannot be identified from the $C_{p}$-time profile alone (section 3.8 ).

\subsection{Global middle-out approach (system parameter estimation)}

In PBPK modelling, it is preferable to use experimentally measured physiological parameters. However, some of the physiological parameters are not available. In such a case, a global middle-out approach has been used to back estimate a physiological parameter from the multiple PK data of multiple drugs (cf. A local middle-out approach is on a drug-by-drug basis) (section 3.1) [83,90,129,145,167]. In this approach, the following three points are usually carefully considered to ensure parameter identifiability and avoid overfitting: (i) enough number of data covering a wide range of dependent and independent variables, (ii) in vivo PK data that is sensitive to the physiological parameter (and not sensitive to the other parameters), (iii) comparison with directly or indirectly measured values.

For example, the volume of the small intestinal fluid $\left(V_{\mathrm{si}}\right)$ available for drug dissolution has not been clear, because only free water can be directly measured by MRI $[28,29]$. The $V_{\text {si }}$ value available for drug dissolution was back-calculated from the clinical PK data of several low solubility drugs at various dose strengths covering Do $<1$ and Do $>1[83,167]$. The estimated $V_{\mathrm{si}}$ values $(130 \mathrm{~mL}$ [83] and $116 \mathrm{~mL}$ [167]) 
were about 1.5 to 2-fold larger than the average free water volume directly measured by various techniques $[26,28,29]$. $D F$ in Eq. 6 was obtained from the relationship between the clinical $F_{\mathrm{a}}$ and $P_{\text {eff }}$ values of about 20 high solubility drugs [55] (cf. $F_{\mathrm{a}}=1-\exp \left(-2 D F / R_{\mathrm{si}} \times P_{\mathrm{eff}} \times T_{\mathrm{si}}\right)$.

\section{Conclusion: strategy to improve oral absorption physiologically-based pharmacokinetic modelling}

\section{Multidisciplinary collaboration}

A good collaboration of pharmacokinetic, physical chemistry, formulation, and physiology experts is required to improve oral absorption (OA) physiologically-based pharmacokinetic (PBPK) modelling. There are many opportunities for both experimentalists and modellers to take advantage of collaborative works in this field [32].

\section{The critical role of physical chemistry in OA-PBPK modelling}

The importance of physical chemistry in PBPK modelling has often been overlooked (section 2.7). Physical chemistry plays a central role in oral drug absorption [86]. In addition, physical chemistry is also important for hepatic clearance, renal clearance, and tissue distribution (including the brain) [85,168-176]. A recent survey suggested that a poor understanding of physical chemistry is one of the reasons for the prediction failure of OA PBPK modelling [6]. A good understanding of the chemical equilibrium [177], nucleation theory $[115,178]$, and fluid dynamics (including mass transport) $[172,173,179]$ is required in OA PBPK modelling. Fortunately, physical chemists are generally well trained in mathematics and mechanistic modelling. They are familiar with the concept of parameter identifiability.

\section{The critical role of physiological parameters in OA PBPK modelling}

Similarly, an in-depth understanding of gastrointestinal physiology is critically important $[165,180,181]$. Physiological parameters reported in the literature have large variations, for example, in gastric $\mathrm{pH}$, intestinal $\mathrm{pH}$, fluid volumes, bile concentration, and buffer capacity $[27,182]$. It is a combination of experimental uncertainty, true inter-subject variability and true inter-occasion variability. The buffer capacity of compendial dissolution media (phosphate buffer) is markedly higher than the real intestinal fluid (bicarbonate buffer), affecting the dissolution profiles of drugs [61,183-186]. The intestinal fluid volume $\left(V_{\text {si }}\right)$ would be much smaller than originally thought [25-29] (note that a $V_{\text {si }}$ value $(212 \mathrm{~mL}$ ) had already been reported as early as 1957) $[26,27]$. The gastric and intestinal pHs of dogs are significantly different from those in humans [50,187-190].

\section{Harmonization}

In the future, it is desirable to harmonize physiological parameters and model equations for regulatory submission (section 1.2 and 2.1). In addition, drug intrinsic parameters such as $S_{0}, P_{\text {transo, }}$ and $K_{\mathrm{bm}}$ (section 2.1), should be obtained using harmonized procedures [109]. Currently, the prediction characteristics of commercial software products vary from product to product [5]. If two software products predict different outcomes, which one should be used for regulatory purposes? In the field of biopharmaceutics, the procedures and conditions for dissolution tests [191,192], in vitro - in vivo correlation [193,194], the biowaiver scheme [195], etc. have been harmonized and standardized. Similarly, OA PBPK modelling should be harmonized based on proper model evaluation in the future (section 2.4). We may also need a good simulation practice when PBPK modelling is used to waive a clinical study $[35,36]$. 
Not to be lost in modelling and simulation

"The greatest enemy of knowledge is not ignorance; it is the illusion of knowledge"

Daniel Boorstin/ Stephen Hawking

When the "bottom-up" simulation deviates from the clinical data, simply press the "optimize" button and the monitor will display a simulation curve that exactly matches the clinical plasma concentration $\left(C_{p}\right)$ time data. If we continue publishing this as a successful "prediction", it will eventually cause the illusion of a prediction paradise.

We must exert extreme caution not to be lost in modelling and simulation. Model equations, physiological parameters, and drug parameters must be disclosed to ensure proper peer-review and reproducibility. A systematic evaluation of predictive power is required to avoid publication bias. When a middle-out approach is pursued, the fitted $C_{p}$ - time curve must be labelled as "fitted" (NOT "predicted"). Parameter identifiability should be carefully considered. The optimized model must be validated using independent clinical PK data of the context of use. Finally, and most importantly, pharmacokinetic, physical chemistry, formulation, and physiology experts should work together so that not to get lost in modelling and simulation.

As mentioned in the introduction, when used correctly, OA PBPK modeling will be an excellent tool for understanding and predicting the oral absorption of a drug. This article will hopefully enhance the science of OA PBPK modelling in the future.

\section{Acknowledgement}

I would like to thank Prof. Kin Tam and Prof. Zoran Mandić for giving me this opportunity to write this article. I would like to thank Dr. Alex Avdeef, Dr. Zahari Vinarov, and Prof. Shinji Yamashita for their valuable and encouraging comments. This article is inspired by the discussions in the Consortium of Biopharmaceutical Tools (CoBiTo) project in Consortium of Pharmaceutical Technology, Research Center for Drug Discovery and Development Science at Ritsumeikan University. The author would like to thank all CoBiTo members, especially Mr. Naoya Matsumura, Mr. Yoshiyuki Akiyama, Ms. Asami Ono, and Mr. Shun Hayashi. The CoBiTo members are from Asahi Kasei Pharma Corporation, Daiichi Sankyo Company, Limited, Daiichi Sankyo RD Novare Co., Ltd., Japan Machinery Co., Ltd., Japan Tobacco Inc., Mitsubishi Tanabe Pharma Corporation, Nippon Boehringer Ingelheim Co., Ltd., Ono Pharmaceutical Co., Ltd., Ritsumeikan University, Sawai Pharmaceutical Co., Ltd., Shionogi \& Co., Ltd., Sumitomo Dainippon Pharma Co., Ltd., and Towa Pharmaceutical Co., Ltd. (in alphabetical order).

\section{Conflict of interest}

Kiyohiko Sugano is the founder of BioavailabilityDesign LLC.

\section{References}

[1] H. Van De Waterbeemd, E. Gifford. ADMET in silico modelling: towards prediction paradise? Nat. Rev. Drug Discov. 2 (2003) 192-204.

[2] A.S. Darwich, A. Margolskee, X. Pepin, L. Aarons, A. Galetin, A. Rostami-Hodjegan, S. Carlert, M. Hammarberg, C. Hilgendorf, P. Johansson, E. Karlsson, D. Murphy, C. Tannergren, H. Thörn, M. Yasin, F. Mazuir, O. Nicolas, S. Ramusovic, C. Xu, S.M. Pathak, T. Korjamo, J. Laru, J. Malkki, S. Pappinen, J. Tuunainen, J. Dressman, S. Hansmann, E. Kostewicz, H. He, T. Heimbach, F. Wu, C. Hoft, Y. Pang, M.B. 
Bolger, E. Huehn, V. Lukacova, J.M. Mullin, K.X. Szeto, C. Costales, J. Lin, M. McAllister, S. Modi, C. Rotter, M. Varma, M. Wong, A. Mitra, J. Bevernage, J. Biewenga, A. Van Peer, R. Lloyd, C. Shardlow, P. Langguth, I. Mishenzon, M.A. Nguyen, J. Brown, H. Lennernäs, B. Abrahamsson. IMI - Oral biopharmaceutics tools project - Evaluation of bottom-up PBPK prediction success part 3: Identifying gaps in system parameters by analysing In Silico performance across different compound classes. Eur. J. Pharm. Sci. 96 (2017) 626-642.

[3] E. Sjögren, H. Thörn, C. Tannergren. Reply to 'Comment on "In Silico Modeling of Gastrointestinal Drug Absorption: Predictive Performance of Three Physiologically Based Absorption Models." Mol. Pharm. 14 (2017) 340-343.

[4] E. Sjögren, H. Thorn, C. Tannergren. In silico modeling of gastrointestinal drug absorption: predictive performance of three physiologically based absorption models. Mol. Pharm. 13 (2016) 1763-1778.

[5] N. Matsumura, S. Hayashi, Y. Akiyama, A. Ono, S. Funaki, N. Tamura, T. Kimoto, M. Jiko, Y. Haruna, A. Sarashina, others. Prediction characteristics of oral absorption simulation software evaluated using structurally diverse low-solubility drugs. J. Pharm. Sci. 109 (2020) 1403-1416.

[6] A. Ahmad, X. Pepin, L. Aarons, Y. Wang, A.S. Darwich, J.M. Wood, C. Tannergren, E. Karlsson, C. Patterson, $\mathrm{H}$. Thörn, others. IMI--Oral biopharmaceutics tools project--Evaluation of bottom-up PBPK prediction success part 4: Prediction accuracy and software comparisons with improved data and modelling strategies. Eur. J. Pharm. Biopharm. (2020).

[7] F.L. Wood, J.B. Houston, D. Hallifax. Clearance prediction methodology needs fundamental improvement: Trends common to rat and human hepatocytes/microsomes and implications for experimental methodology. Drug Metab. Dispos. 45 (2017) 1178-1188.

[8] F.L. Wood, J.B. Houston, D. Hallifax. Importance of the unstirred water layer and hepatocyte membrane integrity in vitro for quantification of intrinsic metabolic clearance. Drug Metab. Dispos. 46 (2018) 268-278.

[9] D.M. Mudie, N. Samiei, D.J. Marshall, G.E. Amidon, C.A.S. Bergström. Selection of in vivo predictive dissolution media using drug substance and physiological properties. AAPS J. 22 (2020) 34.

[10] C. Kuemmel, Y. Yang, X. Zhang, J. Florian, H. Zhu, M. Tegenge, S.-M. Huang, Y. Wang, T. Morrison, I. Zineh. Consideration of a credibility assessment framework in model-informed drug development: potential application to physiologically-based pharmacokinetic modeling and simulation. CPT Pharmacometrics Syst. Pharmacol. 9 (2020) 21-28.

[11] M. Li, P. Zhao, Y. Pan, C. Wagner. Predictive performance of physiologically based pharmacokinetic models for the effect of food on oral drug absorption: Current status. CPT Pharmacometrics Syst. Pharmacol. 7 (2018) 82-89.

[12] M. Shebley, P. Sandhu, A. Emami Riedmaier, M. Jamei, R. Narayanan, A. Patel, S.A. Peters, V.P. Reddy, M. Zheng, L. de Zwart, M. Beneton, F. Bouzom, J. Chen, Y. Chen, Y. Cleary, C. Collins, G.L. Dickinson, N. Djebli, H.J. Einolf, I. Gardner, F. Huth, F. Kazmi, F. Khalil, J. Lin, A. Odinecs, C. Patel, H. Rong, E. Schuck, P. Sharma, S.P. Wu, Y. Xu, S. Yamazaki, K. Yoshida, M. Rowland. Physiologically Based Pharmacokinetic Model Qualification and Reporting Procedures for Regulatory Submissions: A Consortium Perspective. Clin. Pharmacol. Ther. 104 (2018) 88-110.

[13] U.S.F. and D. Administration. Physiologically Based Pharmacokinetic Analyses - Format and Content. Guidance. (2018).

[14] J. Dibella, J.E. Sager, J. Yu, I. Ragueneau-Majlessi, N. Isoherranen, X. Zhuang, C. Lu, A.B. Pharmaceutica Sinica, European Medicines Agency Committee for Medicinal Products for Human Use, A. Nordmark, U.S. Department of Health and Human Services Food and Drug Administration CDER, E. Medicines Agency. Guideline on the qualification and reporting of physiologically based pharmacokinetic (PBPK) modelling and simulation. Acta Pharm. Sin. B. 44 (2016) 27-29. http://www.ema.europa.eu/docs/en GB/document library/Scientific guideline/2016/07/WC50021 1315.pdf\%0Awww.elsevier.com/locate/apsb\%0Awww.sciencedirect.com\%0Ahttp://dx.doi.org/10.10 16/i.apsb.2016.04.004.

[15] D.L. Sackett, W.M.C. Rosenberg, J.A.M. Gray, R.B. Haynes, W.S. Richardson. Evidence based medicine: what it is and what it isn't. (1996). 
[16] I. Masic, M. Miokovic, B. Muhamedagic. Evidence based medicine--new approaches and challenges. Acta Inform. Medica. 16 (2008) 219.

[17] M. Li, P. Zhao, Y. Pan, C. Wagner. Predictive performance of physiologically based pharmacokinetic models for the effect of food on oral drug absorption: current status. CPT Pharmacometrics Syst. Pharmacol. 7 (2018) 82-89.

[18] K. Jaqaman, G. Danuser. Linking data to models: data regression. Nat. Rev. Mol. Cell Biol. 7 (2006) 813-819.

[19] W. Slob, P.H.M. Janssen, J.M. den Hof. Structural identifiability of PBPK models: practical consequences for modeling strategies and study designs. Crit. Rev. Toxicol. 27 (1997) 261-272.

[20] S.A. Peters, H. Dolgos. Requirements to establishing confidence in physiologically based pharmacokinetic (PBPK) models and overcoming some of the challenges to meeting them. Clin. Pharmacokinet. 58 (2019) 1355-1371.

[21] N. Tsamandouras, A. Rostami-Hodjegan, L. Aarons. Combining the "bottom up" and "top down" approaches in pharmacokinetic modelling: Fitting PBPK models to observed clinical data. Br. J. Clin. Pharmacol. 79 (2015) 48-55.

[22] J.W.T. Yates. Structural identifiability of physiologically based pharmacokinetic models. J. Pharmacokinet. Pharmacodyn. 33 (2006) 421-439.

[23] R. Muñoz-Tamayo, L. Puillet, J.-B. Daniel, D. Sauvant, O. Martin, M. Taghipoor, P. Blavy. To be or not to be an identifiable model. Is this a relevant question in animal science modelling? Animal. 12 (2018) 701-712.

[24] R. Bellman, K.J. Åström. On structural identifiability. Math. Biosci. 7 (1970) 329-339.

[25] D.B. Turner, B. Liu, N. Patel, S.M. Pathak, S. Polak, M. Jamei, J. Dressman, A. Rostami-Hodjegan. Comment on "In Silico Modeling of Gastrointestinal Drug Absorption: Predictive Performance of Three Physiologically-Based Absorption Models." Mol. Pharm. 14 (2017) 336-339.

[26] F. Gotch, J. Nadell, I.S. Edelman, others. Gastrointestinal water and electrolytes. IV. The equilibration of deuterium oxide (D 2 O) in gastrointestinal contents and the proportion of total body water (TBW) in the gastrointestinal tract. J. Clin. Invest. 36 (1957) 289-296.

[27] E.L. McConnell, H.M. Fadda, A.W. Basit. Gut instincts: Explorations in intestinal physiology and drug delivery. Int. J. Pharm. 364 (2008) 213-226.

[28] D.M. Mudie, G.L. Amidon, G.E. Amidon, K. Murray, C.L. Hoad, S.E. Pritchard, P.A. Gowland, M.C. Garnett, R.C. Spiller, L. Marciani. Quantification of gastrointestinal liquid volumes and distribution following a $240 \mathrm{~mL}$ dose of water in the fasted state. Mol. Pharm. 11 (2014) 3039-3047.

[29] C. Schiller, C.P. Fröhlich, T. Giessmann, W. Siegmund, H. Mönnikes, N. Hosten, W. Weitschies. Intestinal fluid volumes and transit of dosage forms as assessed by magnetic resonance imaging. Aliment. Pharmacol. Ther. 22 (2005) 971-979.

[30] F.L. Wood, J.B. Houston, D. Hallifax. Clearance prediction methodology needs fundamental improvement: Trends common to rat and human hepatocytes/microsomes and implications for experimental methodology. Drug Metab. Dispos. 45 (2017) 1178-1188.

[31] H. Lennernäs. Animal data: The contributions of the Ussing Chamber and perfusion systems to predicting human oral drug delivery in vivo. Adv. Drug Deliv. Rev. 59 (2007) 1103-1120.

[32] M. Banwarth-Kuhn, S. Sindi. How and why to build a mathematical model: A case study using prion aggregation. J. Biol. Chem. 295 (2020) 5022-5035.

[33] J.W.T. Yates, R.D.O. Jones, M. Walker, S.Y.A. Cheung. Structural identifiability and indistinguishability of compartmental models. Expert Opin. Drug Metab. Toxicol. 5 (2009) 295-302.

[34] C. Cobelli, J.J. Distefano 3rd. Parameter and structural identifiability concepts and ambiguities: a critical review and analysis. Am. J. Physiol. Integr. Comp. Physiol. 239 (1980) R7--R24.

[35] K. Sugano. Introduction to computational oral absorption simulation. Expert Opin. Drug Metab. Toxicol. 5 (2009). 
[36] K. Sugano. Biopharmaceutics Modeling and Simulations: Theory, Practice, Methods, and Applications, 2012.

[37] L. Kuepfer, C. Niederalt, T. Wendl, J.-F. Schlender, S. Willmann, J. Lippert, M. Block, T. Eissing, D. Teutonico. Applied concepts in PBPK modeling: how to build a PBPK/PD model. CPT Pharmacometrics Syst. Pharmacol. 5 (2016) 516-531.

[38] K. Sugano. Emerging Full Mechanistic Physiologically Based Modeling, 2014.

[39] E.G. Rippie, D.J. Lamb, P.W. Romig. Solubilization of weakly acidic and basic drugs by aqueous solutions of polysorbate 80. J. Pharm. Sci. 53 (1964) 1346-1348.

[40] J. Jinno, D. Oh, J.R. Crison, G.L. Amidon. Dissolution of ionizable water-insoluble drugs: The combined effect of pH and surfactant. J. Pharm. Sci. 89 (2000) 268-274.

[41] A.A. Noyes, W.R. Whitney. The rate of solution of solid substances in their own solutions. J. Am. Chem. Soc. 19 (1897) 930-934.

[42] K.G. Mooney, M.A. Mintun, K.J. Himmelstein, V.J. Stella. Dissolution kinetics of carboxylic acids I: effect of pH under unbuffered conditions. J. Pharm. Sci. 70 (1981) 13-22.

[43] K.G. Mooney, M.A. Mintun, K.J. Himmelstein, V.J. Stella. Dissolution kinetics of carboxylic acids II: Effect of buffers. J. Pharm. Sci. 70 (1981) 22-32.

[44] A. Okazaki, T. Mano, K. Sugano. Theoretical dissolution model of poly-disperse drug particles in biorelevant media. J. Pharm. Sci. 97 (2008).

[45] R.J. Hintz, K.C. Johnson. The effect of particle size distribution on dissolution rate and oral absorption. Int. J. Pharm. 51 (1989) 9-17.

[46] J. Wang, D.R. Flanagan. General solution for diffusion-controlled dissolution of spherical particles. 1. Theory. J. Pharm. Sci. 88 (1999) 731-738.

[47] K. Sugano. Theoretical comparison of hydrodynamic diffusion layer models used for dissolution simulation in drug discovery and development. Int. J. Pharm. 363 (2008) 73-77.

[48] S.S. Ozturk, B.O. Palsson, J.B. Dressman. Dissolution of lonizable Drugs in Buffered and Unbuffered Solutions. Pharm. Res. 5 (1988) 272-282.

[49] K. Sugano. Estimation of effective intestinal membrane permeability considering bile micelle solubilisation. Int. J. Pharm. 368 (2009) 116-122.

[50] Y. Akiyama, T. Kimoto, H. Mukumoto, S. Miyake, S. Ito, T. Taniguchi, Y. Nomura, N. Matsumura, T. Fujita, K. Sugano. Prediction accuracy of mechanism-based oral absorption model for dogs. J. Pharm. Sci. 108 (2019) 2728-2736.

[51] Y. Akiyama, S. Ito, T. Fujita, K. Sugano. Prediction of negative food effect induced by bile micelle binding on oral absorption of hydrophilic cationic drugs. Eur. J. Pharm. Sci. (2020) 105543.

[52] K. Sugano. Theoretical investigation of passive intestinal membrane permeability using Monte Carlo method to generate drug-like molecule population. Int. J. Pharm. 373 (2009).

[53] K. Sugano, M. Kataoka, C. da Costa Mathews, S. Yamashita. Prediction of food effect by bile micelles on oral drug absorption considering free fraction in intestinal fluid. Eur. J. Pharm. Sci. 40 (2010).

[54] D. Pade, M. Jamei, A. Rostami-Hodjegan, D.B. Turner. Application of the MechPeff model to predict passive effective intestinal permeability in the different regions of the rodent small intestine and colon. Biopharm. Drug Dispos. 38 (2017) 94-114.

[55] K. Sugano. Estimation of effective intestinal membrane permeability considering bile micelle solubilisation. Int. J. Pharm. 368 (2009).

[56] A. Dokoumetzidis, P. Macheras. A century of dissolution research: From Noyes and Whitney to the Biopharmaceutics Classification System. Int. J. Pharm. 321 (2006) 1-11.

[57] M. Jamei, B. Abrahamsson, J. Brown, J. Bevernage, M.B. Bolger, T. Heimbach, E. Karlsson, E. Kotzagiorgis, A. Lindahl, M. McAllister, others. Current status and future opportunities for incorporation of dissolution data in PBPK modeling for pharmaceutical development and regulatory applications: OrBiTo consortium commentary. Eur. J. Pharm. Biopharm. 155 (2020) 55-68. 
[58] M. McAllister. Dynamic dissolution: A step closer to predictive dissolution testing? Mol. Pharm. 7 (2010) 1374-1387.

[59] B.J. Krieg, S.M. Taghavi, G.L. Amidon, G.E. Amidon. In vivo predictive dissolution: Transport analysis of the CO2, bicarbonate in vivo buffer system. J. Pharm. Sci. 103 (2014) 3473-3490.

[60] H. Lennernäs, A. Lindahl, A. Van Peer, C. Ollier, T. Flanagan, R. Lionberger, A. Nordmark, S. Yamashita, L. Yu, G.L. Amidon, V. Fischer, E. Sjögren, P. Zane, M. McAllister, B. Abrahamsson. In vivo predictive dissolution (IPD) and biopharmaceutical modeling and simulation: Future use of modern approaches and methodologies in a regulatory context. Mol. Pharm. 14 (2017) 1307-1314.

[61] B.J. Krieg, S.M. Taghavi, G.L. Amidon, G.E. Amidon. In Vivo Predictive Dissolution: Comparing the Effect of Bicarbonate and Phosphate Buffer on the Dissolution of Weak Acids and Weak Bases. J. Pharm. Sci. 104 (2015) 2894-2904.

[62] G. Shmueli, others. To explain or to predict? Stat. Sci. 25 (2010) 289-310.

[63] M. Weisberg. Simulation and Similarity: Using Models to Understand the World, OUP USA, 2013. https://books.google.co.jp/books?id=rDu5e532mloC.

[64] M. Weisberg. Simulation and Similarity: Using Models to Understand the World, OUP USA, 2013.

[65] D. Orrell. Apollo's Arrow, HarperCollins, 2008. https://books.google.co.jp/books?id=snMPHwAACAAJ.

[66] A. Hisaka, Y. Ohno, T. Yamamoto, H. Suzuki. Theoretical considerations on quantitative prediction of drug-drug interactions. Drug Metab. Pharmacokinet. 25 (2010) 48-61.

[67] M. Tod, P.B. Pierrillas, L. Bourguignon, S. Goutelle. Comparison of the static in vivo approach to a physiologically based pharmacokinetic approach for metabolic drug--drug interactions prediction. Int. J. Pharmacokinet. 1 (2016) 25-34.

[68] E.I. Ette, P.J. Williams, E.I. Ette, P.J. Williams. Pharmacometrics: the science of quantitative pharmacology / edited by Ene I. Ette, Anoixis Corporation, Paul J. Williams, University of the Pacific and Anoixis Corporation., Wiley-Interscience, Hoboken, New Jersey, 2007.

[69] K. Yamaoka, T. Nakagawa, T. Uno. Application of Akaike's information criterion (AIC) in the evaluation of linear pharmacokinetic equations. J. Pharmacokinet. Biopharm. 6 (1978) 165-175.

[70] R. Takano, K. Furumoto, K. Shiraki, N. Takata, Y. Hayashi, Y. Aso, S. Yamashita. Rate-limiting steps of oral absorption for poorly water-soluble drugs in dogs; prediction from a miniscale dissolution test and a physiologically-based computer simulation. Pharm. Res. 25 (2008) 2334-2344.

[71] L.X. Yu. An integrated model for determining causes of poor oral drug absorption. Pharm. Res. 16 (1999) 1883-1887.

[72] K. Sugano, K. Terada. Rate- and Extent-Limiting Factors of Oral Drug Absorption: Theory and Applications. J. Pharm. Sci. 104 (2015).

[73] K. Sugano, Y. Nabuchi, M. Machida, Y. Aso. Prediction of human intestinal permeability using artificial membrane permeability. Int. J. Pharm. 257 (2003).

[74] A. Avdeef, K.Y. Tam. How well can the caco-2/madin-darby canine kidney models predict effective human jejunal permeability? J. Med. Chem. 53 (2010) 3566-3584.

[75] A.E. Riedmaier, K. DeMent, J. Huckle, P. Bransford, C. Stillhart, R. Lloyd, R. Alluri, S. Basu, Y. Chen, V. Dhamankar, others. Use of Physiologically Based Pharmacokinetic (PBPK) Modeling for Predicting Drug-Food Interactions: an Industry Perspective. AAPS J. 22 (2020) 1-15.

[76] S. J.E., Y. J., R.-M. I., I. N. Physiologically based pharmacokinetic (PBPK) modeling and simulation approaches: A systematic review of published models, applications, and model verification. Drug Metab. Dispos. 43 (2015) 1823-1837.

[77] V.P. Shah, G.L. Amidon. G.L. Amidon, H. Lennernas, V.P. Shah, and J.R. Crison. A Theoretical Basis for a Biopharmaceutic Drug Classification: The Correlation of In Vitro Drug Product Dissolution and In Vivo Bioavailability, Pharm Res 12, 413-420, 1995-Backstory of BCS. AAPS J. 16 (2014) 894-898.

[78] A. Margolskee, A.S. Darwich, X. Pepin, L. Aarons, A. Galetin, A. Rostami-Hodjegan, S. Carlert, M. Hammarberg, C. Hilgendorf, P. Johansson, E. Karlsson, D. Murphy, C. Tannergren, H. Thörn, M. Yasin, F. Mazuir, O. Nicolas, S. Ramusovic, C. Xu, S.M. Pathak, T. Korjamo, J. Laru, J. Malkki, S. Pappinen, J. 
Tuunainen, J. Dressman, S. Hansmann, E. Kostewicz, H. He, T. Heimbach, F. Wu, C. Hoft, L. Laplanche, Y. Pang, M.B. Bolger, E. Huehn, V. Lukacova, J.M. Mullin, K.X. Szeto, C. Costales, J. Lin, M. McAllister, S. Modi, C. Rotter, M. Varma, M. Wong, A. Mitra, J. Bevernage, J. Biewenga, A. Van Peer, R. Lloyd, C. Shardlow, P. Langguth, I. Mishenzon, M.A. Nguyen, J. Brown, H. Lennernäs, B. Abrahamsson. IMI Oral biopharmaceutics tools project - Evaluation of bottom-up PBPK prediction success part 2: An introduction to the simulation exercise and overview of results. Eur. J. Pharm. Sci. 96 (2017) 610-625.

[79] N. Matsumura, A. Ono, Y. Akiyama, T. Fujita, K. Sugano. Bottom-Up Physiologically Based Oral Absorption Modeling of Free Weak Base Drugs. Pharmaceutics. 12 (2020) 844.

[80] M.V.S. Varma, R.S. Obach, C. Rotter, H.R. Miller, G. Chang, S.J. Steyn, A. El-Kattan, M.D. Troutman. Physicochemical space for optimum oral bioavailability: contribution of human intestinal absorption and first-pass elimination. J. Med. Chem. 53 (2010) 1098-1108.

[81] R. Takano, K. Sugano, A. Higashida, Y. Hayashi, M. Machida, Y. Aso, S. Yamashita. Oral absorption of poorly water-soluble drugs: Computer simulation of fraction absorbed in humans from a miniscale dissolution test. Pharm. Res. 23 (2006) 1144-1156.

[82] R. Takano, K. Sugano, A. Higashida, Y. Hayashi, M. Machida, Y. Aso, S. Yamashita. Oral absorption of poorly water-soluble drugs: Computer simulation of fraction absorbed in humans from a miniscale dissolution test. Pharm. Res. 23 (2006).

[83] K. Sugano. Fraction of a dose absorbed estimation for structurally diverse low solubility compounds. Int. J. Pharm. 405 (2011).

[84] N. Hisada, R. Takano, N. Takata, K. Shiraki, T. Ueto, S. Tanida, M. Kataoka, S. Yamashita. Characterizing the dissolution profiles of supersaturable salts, cocrystals, and solvates to enhance in vivo oral absorption. Eur. J. Pharm. Biopharm. 103 (2016) 192-199.

[85] K. Sugano, M. Kansy, P. Artursson, A. Avdeef, S. Bendels, L. Di, G.F. Ecker, B. Faller, H. Fischer, G. Gerebtzoff, H. Lennernaes, F. Senner. Coexistence of passive and carrier-mediated processes in drug transport. Nat. Rev. Drug Discov. 9 (2010).

[86] K. Sugano. Biopharmaceutics Modeling and Simulations: Theory, Practice, Methods, and Applications, Wiley, n.d.

[87] P. Wils, A. Warnery, V. Phung-Ba, S. Legrain, D. Scherman. High lipophilicity decreases drug transport across intestinal epithelial cells. J. Pharmacol. Exp. Ther. 269 (1994) 654-658.

[88] G. Krishna, K.J. Chen, C.C. Lin, A.A. Nomeir. Permeability of lipophilic compounds in drug discovery using in-vitro human absorption model, Caco-2. Int. J. Pharm. 222 (2001) 77-89.

[89] R. Saitoh, K. Sugano, N. Takata, T. Tachibana, A. Higashida, Y. Nabuchi, Y. Aso. Correction of permeability with pore radius of tight junctions in Caco-2 monolayers improves the prediction of the dose fraction of hydrophilic drugs absorbed by humans. Pharm. Res. 21 (2004).

[90] K. Sugano, N. Takata, M. Machida, K. Saitoh, K. Terada. Prediction of passive intestinal absorption using bio-mimetic artificial membrane permeation assay and the paracellular pathway model. Int. J. Pharm. 241 (2002).

[91] G.E. Amidon, W.I. Higuchi, N.F.H. Ho. Theoretical and experimental studies of transport of micellesolubilized solutes. J. Pharm. Sci. 71 (1982) 77-84.

[92] T. YAMAGUCHI, C. IKEDA, Y. SEKINE. Intestinal Absorption of \$ $\beta \$$-Adrenergic Blocking Agent Nadolol. I.: Comparison of Absorption Behaivor of Nadolol with Those of Other $\$ \beta \$$-Blocking Agents in Rats. Chem. Pharm. Bull. 34 (1986) 3362-3369.

[93] F.G.J. Poelma, R. Breäs, J.J. Tukker, D.J.A. Crommelin. Intestinal Absorption of Drugs. The Influence of Mixed Micelles on on the Disappearance Kinetics of Drugs from the Small Intestine of the Rat. J. Pharm. Pharmacol. 43 (1991) 317-324.

[94] F. Ingels, B. Beck, M. Oth, P. Augustijns. Effect of simulated intestinal fluid on drug permeability estimation across Caco-2 monolayers. Int. J. Pharm. 274 (2004) 221-232.

[95] A.T. Heikkinen, J. Mönkkönen, T. Korjamo. Determination of permeation resistance distribution in in vitro cell monolayer permeation experiments. Eur. J. Pharm. Sci. 40 (2010) 132-142. 
[96] F.A. Wilson, V.L. Sallee, J.M. Dietschy. Unstirred water layers in intestine: rate determinant of fatty acid absorption from micellar solutions. Science (80-. ). 174 (1971) 1031-1033.

[97] U. Fagerholm, H. Lennernäs. Experimental estimation of the effective unstirred water layer thickness in the human jejunum, and its importance in oral drug absorption. Eur. J. Pharm. Sci. 3 (1995) 247253.

[98] K.A. Youdim, A. Avdeef, N.J. Abbott. In vitro trans-monolayer permeability calculations: often forgotten assumptions. Drug Discov. Today. 8 (2003) 997-1003.

[99] A. Olivares-Morales, H. Lennernäs, L. Aarons, A. Rostami-Hodjegan. Translating human effective jejunal intestinal permeability to surface-dependent intrinsic permeability: a pragmatic method for a more mechanistic prediction of regional oral drug absorption. AAPS J. 17 (2015) 1177-1192.

[100] K. Sugano, M. Kataoka, C. da Costa Mathews, S. Yamashita. Prediction of food effect by bile micelles on oral drug absorption considering free fraction in intestinal fluid. Eur. J. Pharm. Sci. 40 (2010) 118124.

[101] T. Loftsson, F. Konrádsdóttir, M. Másson. Influence of aqueous diffusion layer on passive drug diffusion from aqueous cyclodextrin solutions through biological membranes. Pharmazie. 61 (2006) 83-89.

[102] A. Dahan, J.M. Miller, A. Hoffman, G.E. Amidon, G.L. Amidon. The solubility--permeability interplay in using cyclodextrins as pharmaceutical solubilizers: mechanistic modeling and application to progesterone. J. Pharm. Sci. 99 (2010) 2739-2749.

[103] R.E. Oliver, A.F. Jones, M. Rowland. What surface of the intestinal epithelium is effectively available to permeating drugs? J. Pharm. Sci. 87 (1998) 634-639.

[104] A. Avdeef, P.E. Nielsen, O. Tsinman. PAMPA - A drug absorption in vitro model: 11. Matching the in vivo unstirred water layer thickness by individual-well stirring in microtitre plates. Eur. J. Pharm. Sci. 22 (2004) 365-374.

[105] M. Vertzoni, E. Pastelli, D. Psachoulias, L. Kalantzi, C. Reppas. Estimation of intragastric solubility of drugs: In what medium? Pharm. Res. 24 (2007) 909-917.

[106] A.T.M. Serajuddin, C.I. Jarowski. Effect of diffusion layer pH and solubility on the dissolution rate of pharmaceutical bases and their hydrochloride salts I: phenazopyridine. J. Pharm. Sci. 74 (1985) 142147.

[107] J. Al-Gousous, N. Salehi, G.E. Amidon, R.M. Ziff, P. Langguth, G.L. Amidon. Mass Transport Analysis of Bicarbonate Buffer: Effect of the $\mathrm{CO} 2-\mathrm{H} 2 \mathrm{CO} 3$ Hydration-Dehydration Kinetics in the Fluid Boundary Layer and the Apparent Effective p Ka Controlling Dissolution of Acids and Bases. Mol. Pharm. 16 (2019) 2626-2635.

[108] K. Sugano, A. Okazaki, S. Sugimoto, S. Tavornvipas, A. Omura, T. Mano. Solubility and Dissolution Profile Assessment in Drug Discovery. Drug Metab. Pharmacokinet. 22 (2007) 225-254.

[109] A. Ono, N. Matsumura, T. Kimoto, Y. Akiyama, S. Funaki, N. Tamura, S. Hayashi, Y. Kojima, M. Fushimi, $\mathrm{H}$. Sudaki, others. Harmonizing solubility measurement to lower inter-laboratory variance--progress of consortium of biopharmaceutical tools (CoBiTo) in Japan. ADMET DMPK 7 (2019) 183-195. https://doi.org/10.5599/admet.704.

[110] A. Avdeef. Suggested Improvements for Measurement of Equilibrium Solubility-pH of lonizable Drugs. ADMET DMPK 3 (2015) 84-109. https://doi.org/10.5599/admet.3.2.193.

[111] A. Avdeef, E. Fuguet, A. Llinàs, C. Ràfols, E. Bosch, G. Völgyi, T. Verbić, E. Boldyreva, K. Takács-Novák. Equilibrium solubility measurement of ionizable drugs - consensus recommendations for improving data quality. ADMET DMPK 4 (2016) 117. https://doi.org/10.5599/admet.4.2.292.

[112] T. Uekusa, K. Sugano. Effect of buffer capacity on dissolution and supersaturation profiles of poorly soluble drug salt, in: APSJT Annu. Meet., Toyama, 2019: p. 236.

[113] J. Oki, D. Watanabe, T. Uekusa, K. Sugano. Mechanism of Supersaturation Suppression in Dissolution Process of Acidic Drug Salt. Mol. Pharm. 16 (2019) 1669-1677.

[114] K. Sugano. A simulation of oral absorption using classical nucleation theory. Int. J. Pharm. 378 (2009) 142-145. 
[115] S. Ozaki. Population Balance Model for Simulation of the Supersaturation-Precipitation Behavior of Drugs in Supersaturable Solid Forms. J. Pharm. Sci. 108 (2019) 260-267.

[116] L. Lindfors, S. Forssén, J. Westergren, U. Olsson. Nucleation and crystal growth in supersaturated solutions of a model drug. J. Colloid Interface Sci. 325 (2008) 404-413.

[117] S. Ozaki, T. Minamisono, T. Yamashita, T. Kato, I. Kushida. Supersaturation--nucleation behavior of poorly soluble drugs and its impact on the oral absorption of drugs in thermodynamically high-energy forms. J. Pharm. Sci. 101 (2012) 214-222.

[118] M. Warzecha, R. Guo, R.M. Bhardwaj, S.M. Reutzel-Edens, S.L. Price, D.A. Lamprou, A.J. Florence. Direct observation of templated two-step nucleation mechanism during olanzapine hydrate formation. Cryst. Growth Des. 17 (2017) 6382-6393.

[119] P. Jakubiak, B. Wagner, H.P. Grimm, J. Petrig-Schaffland, F. Schuler, R. Alvarez-Sánchez. Development of a Unified Dissolution and Precipitation Model and Its Use for the Prediction of Oral Drug Absorption. Mol. Pharm. 13 (2016) 586-598.

[120] A. Llinas, I. Oprisiu, A. Avdeef. Findings of the Second Challenge to Predict Aqueous Solubility. J. Chem. Inf. Model. (2020).

[121] K. Takács-Novák, M. Urac, P. Horváth, G. Völgyi, B.D. Anderson, A. Avdeef. Equilibrium solubility measurement of compounds with low dissolution rate by Higuchi's Facilitated Dissolution Method. A validation study. Eur. J. Pharm. Sci. 106 (2017) 133-141.

[122] N. Sun, A. Avdeef. Biorelevant pK a $\left(37^{\circ} \mathrm{C}\right)$ predicted from the $2 \mathrm{D}$ structure of the molecule and its $\mathrm{pK}$ a at $25^{\circ}$ C. J. Pharm. Biomed. Anal. 56 (2011) 173-182.

[123] A. Avdeef. Solubility Temperature Dependence Predicted from 2D Structure. ADMET DMPK. 3 (2015) 298-344. https://doi.org/10.5599/admet.3.4.259.

[124] S.S. Hate, S.M. Reutzel-Edens, L.S. Taylor. Absorptive Dissolution Testing of Supersaturating Systems: Impact of Absorptive Sink Conditions on Solution Phase Behavior and Mass Transport. Mol. Pharm. 14 (2017) 4052-4063.

[125] D.J. Good, R.H. Naír. Solubility advantage of pharmaceutical cocrystals. Cryst. Growth Des. 9 (2009) 2252-2264.

[126] M. Omori, T. Watanabe, T. Uekusa, J. Oki, D. Inoue, K. Sugano. Effect of coformer and polymer on particle surface solution-mediated phase transformation of cocrystal in aqueous media. Mol. Pharm. (2020).

[127] B. Van Eerdenbrugh, G. Van Den Mooter, P. Augustijns, J. Vermant, J.A. Martens, L. Froyen, J. Van Humbeeck. Solubility increases associated with crystalline drug nanoparticles: Methodologies and significance. Mol. Pharm. 7 (2010) 1858-1870.

[128] S.B. Murdande, D.A. Shah, R.H. Dave. Impact of nanosizing on solubility and dissolution rate of poorly soluble pharmaceuticals. J. Pharm. Sci. 104 (2015) 2094-2102.

[129] K. Sugano. Possible reduction of effective thickness of intestinal unstirred water layer by particle drifting effect. Int. J. Pharm. 387 (2010).

[130] J.F. Cuine, C.L. McEvoy, W.N. Charman, C.W. Pouton, G.A. Edwards, H. Benameur, C.J.H. Porter. Evaluation of the impact of surfactant digestion on the bioavailability of danazol after oral administration of lipidic self-emulsifying formulations to dogs. J. Pharm. Sci. 97 (2008) 995-1012.

[131] A.T. Larsen, P. Sassene, A. Müllertz. In vitro lipolysis models as a tool for the characterization of oral lipid and surfactant based drug delivery systems. Int. J. Pharm. 417 (2011) 245-255.

[132] D. Orrell. Apollo's Arrow, HarperCollins, 2008.

[133] S.A. Peters, P.E. Schroeder, N. Giri, H. Dolgos. Evaluation of the use of static and dynamic models to predict drug-drug interaction and its associated variability: Impact on drug discovery and early development. Drug Metab. Dispos. 40 (2012) 1495-1507.

[134] J.B. Dressman, D. Fleisher. Mixing-tank model for predicting dissolution rate control of oral absorption. J. Pharm. Sci. 75 (1986) 109-116. 
[135] E. Nicolaides, M. Symillides, J.B. Dressman, C. Reppas. Biorelevant dissolution testing to predict the plasma profile of lipophilic drugs after oral administration. Pharm. Res. 18 (2001) 380-388.

[136] C. Wagner, E. Jantratid, F. Kesisoglou, M. Vertzoni, C. Reppas, J. B. Dressman. Predicting the oral absorption of a poorly soluble, poorly permeable weak base using biorelevant dissolution and transfer model tests coupled with a physiologically based pharmacokinetic model. Eur. J. Pharm. Biopharm. 82 (2012) 127-138.

[137] S. Chakraborty, L. Yadav, D. Aggarwal. Prediction of in vivo drug performance using in vitro dissolution coupled with STELLA: a study with selected drug products. Drug Dev. Ind. Pharm. 41 (2015) 1667-1673.

[138] A. Kambayashi, T. Yasuji, J.B. Dressman. Prediction of the precipitation profiles of weak base drugs in the small intestine using a simplified transfer ("dumping") model coupled with in silico modeling and simulation approach. Eur. J. Pharm. Biopharm. 103 (2016) 95-103.

[139] A. Kambayashi, J.B. Dressman. Forecasting gastrointestinal precipitation and oral pharmacokinetics of dantrolene in dogs using an in vitro precipitation testing coupled with in silico modeling and simulation. Eur. J. Pharm. Biopharm. 119 (2017) 107-113.

[140] A. Kambayashi, J.B. Dressman. An in vitro-in silico-in vivo approach to predicting the oral pharmacokinetic profile of salts of weak acids: Case example dantrolene. Eur. J. Pharm. Biopharm. 84 (2013) 200-207.

[141] K. Matsui, Y. Tsume, S. Takeuchi, A. Searls, G.L. Amidon. Utilization of gastrointestinal simulator, an in vivo predictive dissolution methodology, coupled with computational approach to forecast oral absorption of dipyridamole. Mol. Pharm. 14 (2017) 1181-1189.

[142] M. Yamane, K. Matsui, M. Sugihara, Y. Tokunaga. The provisional no-effect threshold of sugar alcohols on oral drug absorption estimated by physiologically based biopharmaceutics model. J. Pharm. Sci. (2020).

[143] A. Kambayashi, T. Kiyota. A Physiologically-based Drug Absorption Modeling for Orally Disintegrating Tablets. Eur. J. Pharm. Biopharm. (2020).

[144] Y. Cao, W.J. Jusko. Applications of minimal physiologically-based pharmacokinetic models. J. Pharmacokinet. Pharmacodyn. 39 (2012) 711-723.

[145] R. Li, H.A. Barton, P.D. Yates, A. Ghosh, A.C. Wolford, K.A. Riccardi, T.S. Maurer. A "middle-out" approach to human pharmacokinetic predictions for OATP substrates using physiologically-based pharmacokinetic modeling. J. Pharmacokinet. Pharmacodyn. 41 (2014) 197-209.

[146] D.-M. Oh, R.L. Curl, G.L. Amidon. Estimating the fraction dose absorbed from suspensions of poorly soluble compounds in humans: a mathematical model. Pharm. Res. 10 (1993) 264-270.

[147] H. Lennernäs, C.G. Regårdh. Regional Gastrointestinal Absorption of the Beta-Blocker Pafenolol in the Rat and Intestinal Transit Rate Determined by Movement of 14C-Polyethylene Glycol (PEG) 4000. Pharm. Res. An Off. J. Am. Assoc. Pharm. Sci. 10 (1993) 130-135.

[148] D. Riethorst, J. Brouwers, J. Motmans, P. Augustijns. Human intestinal fluid factors affecting intestinal drug permeation in vitro. Eur. J. Pharm. Sci. 121 (2018) 338-346.

[149] K. Sugano. Calculation of fraction of dose absorbed: comparison between analytical solution based on one compartment steady state concentration approximation and dynamic seven compartment model. Chem-Bio Informatics J. 9 (2009) 75-93.

[150] M.V.S. Varma, R.S. Obach, C. Rotter, H.R. Miller, G. Chang, S.J. Steyn, A. El-Kattan, M.D. Troutman. Physicochemical space for optimum oral bioavailability: Contribution of human intestinal absorption and first-pass elimination. J. Med. Chem. 53 (2010) 1098-1108.

[151] E. Sjögren, D. Dahlgren, C. Roos, H. Lennernäs. Human in vivo regional intestinal permeability: Quantitation using site-specific drug absorption data. Mol. Pharm. 12 (2015) 2026-2039.

[152] D. Psachoulias, M. Vertzoni, K. Goumas, V. Kalioras, S. Beato, J. Butler, C. Reppas. Precipitation in and supersaturation of contents of the upper small intestine after administration of two weak bases to fasted adults. Pharm. Res. 28 (2011) 3145-3158. 
[153] B. Hens, J. Brouwers, M. Corsetti, P. Augustijns. Supersaturation and Precipitation of Posaconazole Upon Entry in the Upper Small Intestine in Humans. J. Pharm. Sci. 105 (2016) 2677-2684.

[154] K.K.H. Chan, F. Langenbucher, M. Gibaldi. Evaluation of in vivo drug release by numerical deconvolution using oral solution data as weighting function. J. Pharm. Sci. 76 (1987) 446-450.

[155] S. Clarysse, J. Brouwers, J. Tack, P. Annaert, P. Augustijns. Intestinal drug solubility estimation based on simulated intestinal fluids: Comparison with solubility in human intestinal fluids. Eur. J. Pharm. Sci. 43 (2011) 260-269.

[156] K.S. Shingo Takezawa, Kikuo Ozawa, Rishichi Mimura. Membrane permeation mechanism of solute in dialyzer. Artif. Organ. 13 (1984) 1460-1467.

[157] A. Avdeef. Leakiness and size exclusion of paracellular channels in cultured epithelial cell monolayersinterlaboratory comparison. Pharm. Res. 27 (2010) 480-489.

[158] M. Kato, K. Chiba, A. Hisaka, M. Ishigami, M. Kayama, N. Mizuno, Y. Nagata, S. Takakuwa, Y. Tsukamoto, K. Ueda, H. Kusuhara, K. Ito, Y. Sugiyama. The Intestinal First-pass Metabolism of Substrates of CYP3A4 and P-glycoprotei-Quantitative Analysis Based on Information from the Literature. Drug Metab. Pharmacokinet. 18 (2004) 365-372.

[159] H. Lennernäs. Intestinal permeability and its relevance for absorption and elimination, 2007.

[160] E. Luzon, K. Blake, S. Cole, A. Nordmark, C. Versantvoort, E.G. Berglund. Physiologically based pharmacokinetic modeling in regulatory decision-making at the European Medicines Agency. Clin. Pharmacol. Ther. 102 (2017) 98-105.

[161] M. Grimstein, Y. Yang, X. Zhang, J. Grillo, S.-M. Huang, I. Zineh, Y. Wang. Physiologically based pharmacokinetic modeling in regulatory science: an update from the US Food and Drug Administration's Office of Clinical Pharmacology. J. Pharm. Sci. 108 (2019) 21-25.

[162] A. Hisaka, Y. Ohno, T. Yamamoto, H. Suzuki. Theoretical Considerations on Quantitative Prediction of Drug-Drug Interactions. Drug Metab. Pharmacokinet. 25 (2010) 48-61.

[163] Y. Ohno, A. Hisaka, H. Suzuki. General framework for the quantitative prediction of CYP3A4-mediated oral drug interactions based on the AUC increase by coadministration of standard drugs. Clin. Pharmacokinet. 46 (2007) 681-696.

[164] A. Hisaka, Y. Ohno, T. Yamamoto, H. Suzuki. Prediction of pharmacokinetic drug-drug interaction caused by changes in cytochrome P450 activity using in vivo information. Pharmacol. Ther. 125 (2010) 230-248.

[165] M. Koziolek, S. Alcaro, P. Augustijns, A.W. Basit, M. Grimm, B. Hens, C.L. Hoad, P. Jedamzik, C.M. Madla, M. Maliepaard, others. The mechanisms of pharmacokinetic food-drug interactions--A perspective from the UNGAP group. Eur. J. Pharm. Sci. 134 (2019) 31-59.

[166] P. Macheras, P. Chryssafidis. Revising Pharmacokinetics of Oral Drug Absorption: I Models Based on Biopharmaceutical/Physiological and Finite Absorption Time Concepts. Pharm. Res. 37 (2020) 1-13.

[167] A. Maharaj, N. Fotaki, A. Edginton. Parameterization of small intestinal water volume using PBPK modeling. Eur. J. Pharm. Sci. 67 (2015) 55-64.

[168] L. Di, P. Artursson, A. Avdeef, L.Z. Benet, J.B. Houston, M. Kansy, E.H. Kerns, H. Lennernäs, D.A. Smith, K. Sugano. The Critical Role of Passive Permeability in Designing Successful Drugs. ChemMedChem. (2020).

[169] K. Utsey, M.S. Gastonguay, S. Russell, R. Freling, M.M. Riggs, A. Elmokadem. Quantification of the Impact of Partition Coefficient Prediction Methods on Physiologically Based Pharmacokinetic Model Output Using a Standardized Tissue Composition. Drug Metab. Dispos. 48 (2020) 903-916.

[170] K. Valko, S. Rava, S. Bunally, S. Anderson. Revisiting the application of Immobilized Artificial Membrane (IAM) chromatography to estimate in vivo distribution properties of drug discovery compounds based on the model of marketed drugs. ADMET DMPK. 8 (2020) 78-97. https://doi.org/10.5599/admet.757.

[171] F. Lombardo, J. Bentzien, G. Berellini, I. Muegge. In silico models of human pk parameters. Prediction of volume of distribution using an extensive data set and a reduced number of parameters. J. Pharm. Sci. (2020). 
[172] D. Hallifax, F.L. Wood. Importance of the unstirred water layer and hepatocyte membrane integrity in vitro for quantification of intrinsic metabolic clearance. Drug Metab. Dispos. 46 (2018) 268-278.

[173] K. Sugano. Aqueous boundary layers related to oral absorption of a drug: From dissolution of a drug to carrier mediated transport and intestinal wall metabolism. Mol. Pharm. 7 (2010) 1362-1373.

[174] L. Di, E.H. Kerns, K. Fan, O.J. McConnell, G.T. Carter. High throughput artificial membrane permeability assay for blood--brain barrier. Eur. J. Med. Chem. 38 (2003) 223-232.

[175] C. Dagenais, A. Avdeef, O. Tsinman, A. Dudley, R. Beliveau. P-glycoprotein deficient mouse in situ blood-brain barrier permeability and its prediction using an in combo PAMPA model. Eur. J. Pharm. Sci. 38 (2009) 121-137.

[176] B. Sinkó, T.M. Garrigues, G.T. Balogh, Z.K. Nagy, O. Tsinman, A. Avdeef, K. Takács-Novák. SkinPAMPA: A new method for fast prediction of skin penetration. Eur. J. Pharm. Sci. 45 (2012) 698-707.

[177] A. Avdeef. Absorption and Drug Development: Solubility, Permeability, and Charge State, Second Edition, John Wiley \& Sons, Inc., 2012.

[178] K. Sugano. A simulation of oral absorption using classical nucleation theory. Int. J. Pharm. 378 (2009).

[179] M. Higuchi, Y. Yoshihashi, K. Tarada, K. Sugano. Minimum rotation speed to prevent coning phenomena in compendium paddle dissolution apparatus. Eur. J. Pharm. Sci. 65 (2014) 74-78.

[180] C. Stillhart, K. Vučićević, P. Augustijns, A.W. Basit, H. Batchelor, T.R. Flanagan, I. Gesquiere, R. Greupink, D. Keszthelyi, M. Koskinen, others. Impact of gastrointestinal physiology on drug absorption in special populations--An UNGAP review. Eur. J. Pharm. Sci. (2020) 105280.

[181] M. Vertzoni, P. Augustijns, M. Grimm, M. Koziolek, G. Lemmens, N. Parrott, C. Pentafragka, C. Reppas, J. Rubbens, J. Van Den Abeele, others. Impact of regional differences along the gastrointestinal tract of healthy adults on oral drug absorption: an UNGAP review. Eur. J. Pharm. Sci. 134 (2019) 153-175.

[182] D.M. Mudie, G.L. Amidon, G.E. Amidon. Physiological parameters for oral delivery and in vitro testing. Mol. Pharm. 7 (2010) 1388-1405.

[183] B. Hens, Y. Tsume, M. Bermejo, P. Paixao, M.J. Koenigsknecht, J.R. Baker, W.L. Hasler, R. Lionberger, J. Fan, J. Dickens, K. Shedden, B. Wen, J. Wysocki, R. Loebenberg, A. Lee, A. Frances, G. Amidon, A. Yu, G. Benninghoff, N. Salehi, A. Talattof, D. Sun, G.L. Amidon. Low Buffer Capacity and Alternating Motility along the Human Gastrointestinal Tract: Implications for in Vivo Dissolution and Absorption of lonizable Drugs. Mol. Pharm. 14 (2017) 4281-4294.

[184] J. Al-Gousous, G.L. Amidon, P. Langguth. Toward Biopredictive Dissolution for Enteric Coated Dosage Forms. Mol. Pharm. 13 (2016) 1927-1936.

[185] J. Al-Gousous, K.X. Sun, D.P. McNamara, B. Hens, N. Salehi, P. Langguth, M. Bermejo, G.E. Amidon, G.L. Amidon. Mass Transport Analysis of the Enhanced Buffer Capacity of the Bicarbonate-CO2 Buffer in a Phase-Heterogenous System: Physiological and Pharmaceutical Significance. Mol. Pharm. 15 (2018) 5291-5301.

[186] T. Uekusa, J. Oki, M. Omori, D. Watanabe, D. Inoue, K. Sugano. Effect of buffer capacity on dissolution and supersaturation profiles of pioglitazone hydrochloride. J. Drug Deliv. Sci. Technol. 55 (2020) 101492.

[187] H. Ogata, N. Aoyagi, N. Kaniwa, A. Ejima, T. Kitaura, T. Ohki, K. Kitamura. Evaluation of beagle dogs as an animal model for bioavailability testing of cinnarizine capsules. Int. J. Pharm. 29 (1986) 121-126.

[188] K. Sagawa, F. Li, R. Liese, S.C. Sutton. Fed and fasted gastric pH and gastric residence time in conscious beagle dogs. J. Pharm. Sci. 98 (2009) 2494-2500.

[189] C.Y. Lui, G.L. Amidon, R.R. Berardi, D. Fleisher, C. Youngberg, J.B. Dressman. Comparison of gastrointestinal $\mathrm{pH}$ in dogs and humans: implications on the use of the beagle dog as a model for oral absorption in humans [published erratum appears in J Pharm Sci 1986 Dec;75(12):1207]. J. Pharm. Sci. 75 (1986) 271-274.

[190] N. Matsumura, Y. Yamaura, J. Katagi, S. Ono, S. Kim, S. Yamashita, K. Sugano. Evaluation of Using Dogs to Predict Fraction of Oral Dose Absorbed in Humans for Poorly Water-Soluble Drugs. J. Pharm. Sci. 107 (2018) 2489-2496. 
[191] R. Solid, O. Dosage. Guidance for Industry Guidance for Industry Dissolution Testing of Immediate. (1997).

[192] V.P. Shah, L.J. Lesko, J. Fan, N. Fleischer, J. Handerson, H. Malinowski, M. Makary, L. Ouderkirk, S. Bay, P. Sathe, G.J.P. Singh, L. lillman, Y. Tsong, R.I. Williams. FDA guidance for industry 1 dissolution testing of immediate release solid oral dosage forms. Dissolution Technol. 4 (1997) 15-22.

[193] Fda. Extended Release Oral Dosage Forms: Development, Evaluation, and Application of In Vitro/In Vivo Correlations. (1997) 1-24. http://www.fda.gov/cder/guidance/index.htm.

[194] Venkata Ramana S. Uppoor. Regulatory perspectives on in vitro (dissolution) /in vivo (bioavailability) correlations. J. Control. Release. 72 (2001) 127-132.

[195] REDC/ADF. Dissolution Testing and Acceptance Criteria for Immediate-Release Solid Oral Dosage Form Drug Products Containing High Solubility Drug Substances Guidance for Industry. Fda. (2018) August-August. 